\title{
Growth rate of Lithium filaments in ceramic electrolytes
}

\author{
S. S. Shishvan ${ }^{\mathrm{a}, \mathrm{b}}$, N.A. Fleck ${ }^{\mathrm{b}}$, R.M. McMeeking ${ }^{\mathrm{c}, \mathrm{d}, \mathrm{e}}$ and V.S. Deshpande ${ }^{\mathrm{b} *}$ \\ ${ }^{a}$ Department of Structural Engineering, University of Tabriz, Tabriz, Iran \\ ${ }^{\mathrm{b}}$ Department of Engineering, University of Cambridge, Cambridge CB2 1PZ, UK \\ ${ }^{\mathrm{c}}$ Department of Mechanical Engineering \& Materials Department, University of California, \\ Santa Barbara CA 93106, USA \\ ${ }^{\mathrm{d}}$ School of Engineering, Aberdeen University, King's College, Aberdeen AB24 3UE, UK \\ e INM - Leibniz Institute for New Materials, Campus D2 2, 66123 Saarbrücken, Germany
}

\begin{abstract}
Lithium-ion batteries with single ion-conductor ceramic electrolytes short-circuit when subjected to charging currents above a critical current density. Here, we analyse the rate at which a lithium $(\mathrm{Li})$ filament (sometimes referred to as a dendrite) will grow from the cathode towards the anode during charging of such batteries. The filament is modelled as a climbing edge dislocation with its growth occurring by $\mathrm{Li}^{+}$flux from the electrolyte into the filament tip at constant chemical potential. The growth rate is set by a balance between the reduction of free-energy at the filament tip and energy dissipation associated with the resistance to the flux of $\mathrm{Li}^{+}$through the filament tip. For charging currents above the critical current density, the filament growth rate increases with decreasing filament tip resistance. Imperfections, such as voids in the Li cathode along the electrolyte/cathode interface, decrease the critical current density but filament growth rates are also lower in these cases as filament growth rates scale with the charging currents. The predictions of the model are in excellent quantitative agreement with measurements and confirm that above the critical current density a filament can traverse the electrolyte in minutes or less. This suggests that initiation of filament growth is the critical step to prevent short-circuiting of the battery.
\end{abstract}

Keywords: Ceramic electrolyte, solid-state battery, Lithium filament, kinetics.

*Corresponding author. E-mail address: vsd@eng.cam.ac.uk 


\section{Introduction}

Ceramic electrolytes are under consideration for use in lithium-ion batteries as, combined with lithium (Li) metal anodes, they have the potential to deliver enhanced safety and higher energy densities compared to liquid electrolyte Li-ion batteries [1,2]. Liquid electrolyte batteries with a Li metal electrode also have the drawback that they suffer from dendrite nucleation and growth from the electrolyte/electrode interfaces. These dendrites can take the form of mossy growth [3], needle-like protrusions or globular structures [4, 5] and grow across the electrolyte to short-circuit the battery. The high stiffness of ceramic electrolytes is expected to suppress dendrite nucleation and growth. However, solid electrolyte batteries too exhibit a critical current density $i_{\mathrm{CCD}}$ and short-circuiting occurs when the cell is charged at currents above the critical value $[6,7]$.

Currently, the most widely investigated ceramic electrolyte is a single-ion conductor electrolyte $\mathrm{Li}_{7} \mathrm{La}_{3} \mathrm{Zr}_{2} \mathrm{O}_{12}$ (LLZO) with a high $\mathrm{Li}^{+}$conductivity. The critical current densities for these electrolytes increase with decreasing interfacial resistance $Z$ between the electrolyte and the $\mathrm{Li}$ electrodes [8] and increasing grain size of the LLZO [9]. The precise morphology of the Li structures (broadly referred to as filaments in this study) that penetrate LLZO is unclear, although there exist some initial observations $[10,11]$ that suggest that filaments are in the form of cracks with parallel-sided flanks, i.e. resemble edge dislocations. More extensive observations are available for $\mathrm{Na}^{+}$/beta-alumina systems [12] where there is clearer evidence of $\mathrm{Na}$ penetration into the beta-alumina electrolytes in an edge dislocation mode. A recent study [13] has classified Li-filled defects in LLZO ranging from crack-like and dislocation-like features to "diffuse damage" where presumably micro-voids within the LLZO are filled with Li.

The majority of existing analyses have modelled dendrites/filaments as pressurised cracks such that the electrical overpotential of $\mathrm{Li}^{+}$in the electrolyte with respect to $\mathrm{Li}^{+}$in the filament is balanced by pressure within the filament [14-17]. Modelling based on this assumption was initiated in the context of the $\mathrm{Na}^{+}$/beta-alumina systems [14] and more recently extended to the Li-based garnet materials. These models, however, significantly over-predict the critical current densities, as first recognised by Feldman and de Jonghe [14]. In a recent study [18], we have modelled filaments as climbing edge dislocations, with the initiation of filament growth occurring by the flux of $\mathrm{Li}^{+}$from the electrolyte into the filament tip at constant chemical potential. This mechanism, in the presence of imperfections such as voids on the electrolyte/cathode interface [19], give predictions of $i_{C C D}$ that are in good agreement with measurements. Moreover, by idealising the filaments as dislocation-like entities, the pressure within the Li filament vanishes at the junction between the filament and the Li electrode. In contrast, previous studies have analysed dendrites/filaments as internally pressurised cracks [14-17] with a pressure $p_{\mathrm{Li}}$. In order for the crack to advance, the internal pressure is unrealistically high: the pressure at the crack mouth (at the base of the Li filament adjacent to the electrode) cannot be supported by the underlying Li electrode. The pressure far exceeds the yield strength of the Li even accounting for size effects [20] in the strength of Li. 
The question remains: what is the growth rate of filaments in $\mathrm{Li} / \mathrm{LLZO} / \mathrm{Li}$ symmetric cells [611]? Unfortunately, direct observations of the growth of filaments in ceramic electrolytes, in contrast to liquid electrolytes [21], are scarce. Inferences may be drawn from the critical current density measurements $[8,9]$. In these experiments, the imposed current was increased stepwise, with the current held fixed for about 30 minutes during each increment. Negligible changes in the applied voltage were observed during each interval of constant current when the current was below $i_{\mathrm{CCD}}$. However, upon imposition of $i_{\mathrm{CCD}}$, the batteries short-circuited within the 30 minute cycle time, suggesting that filament growth over the $1 \mathrm{~mm}$ thick electrolyte occurred in less than 30 minutes, implying an average growth rate of at least $0.5 \mu \mathrm{m} \mathrm{s}^{-1}$. In a recent study [13], direct measurements of filament growth rates are reported over short lengths (on the order of $10-20 \mu \mathrm{m}$ ); the observed growth rate increases from less than $1 \mu \mathrm{m} \mathrm{s}^{-1}$ to more than $10 \mu \mathrm{m} \mathrm{s}^{-1}$ with increasing imposed current. The aim of the present paper is to develop a theoretical framework for the growth rate of filaments as modelled by climbing dislocations, and to predict the sensitivity of filament growth rate to current density and to the presence of defects such as voids on the electrolyte/electrode interface. A list of the mathematical symbols used in the analysis is provided in Table 1.

Table 1 A summary of the parameters of the model.

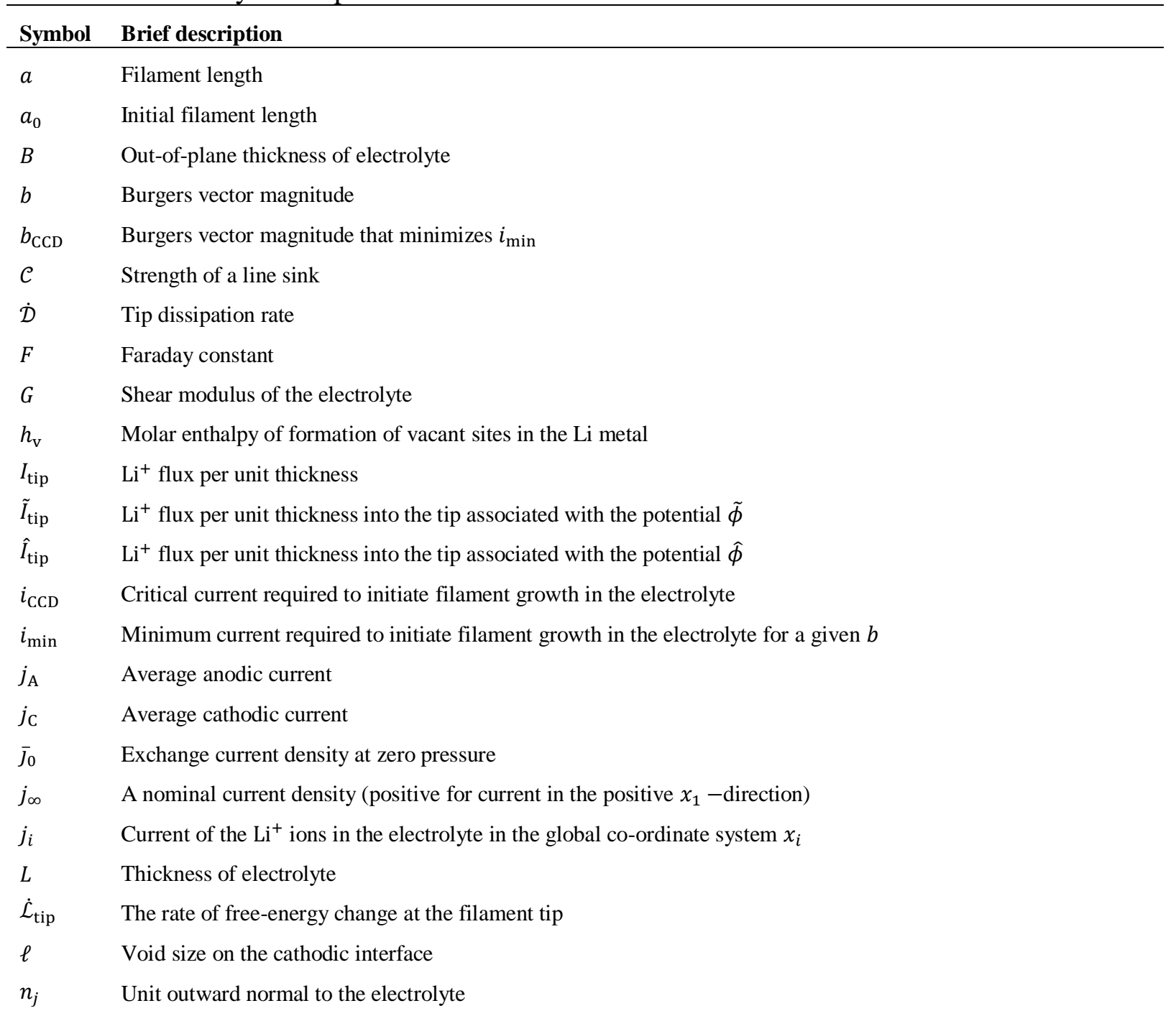




\begin{tabular}{|c|c|}
\hline$R$ & Gas constant \\
\hline$R_{\text {tip }}$ & Tip resistance \\
\hline$r$ & A polar co-ordinate system with the filament tip located at the origin $r=0$ \\
\hline$s_{\text {tip }}$ & Specific entropy of the system defined in the electrolyte at the filament tip \\
\hline$T$ & Temperature \\
\hline $\mathcal{u}$ & Open circuit potential \\
\hline$v \equiv \dot{a}$ & Growing rate of the filament \\
\hline$W$ & Width of electrolyte \\
\hline$x_{i}$ & Global co-ordinate system \\
\hline$Z$ & Interfacial ionic resistance \\
\hline$\beta$ & Butler-Volmer symmetry factor \\
\hline$\gamma_{\mathrm{e} / \mathrm{Li}}$ & Energy per unit area of the electrolyte/Li metal interface \\
\hline$\Delta a$ & An infinitesimal propagation distance of the filament \\
\hline$\Delta \mathcal{L}_{\text {tip }}$ & Free-energy change at the filament tip associated with the filament growth \\
\hline$\eta$ & Overpotential \\
\hline$\hat{\eta}$ & A smooth overpotential \\
\hline$\eta_{\mathrm{c}}$ & Critical filament-tip overpotential \\
\hline$\eta_{\text {tip }}$ & Overpotential of the electrolyte at the filament tip \\
\hline$\theta_{\text {tip }}$ & Occupancy of lattice sites within the metal phase at the filament tip \\
\hline$\kappa$ & Ionic conductivity of the electrolyte \\
\hline$v$ & Poisson's ratio of the electrolyte \\
\hline$\rho_{\mathrm{m}}$ & Theoretical molar density of $\mathrm{Li}$ \\
\hline$\phi$ & Electric potential \\
\hline$\tilde{\phi}$ & Electric potential at location $r$ due to a line sink of strength $\mathcal{C}$ in an infinite medium \\
\hline$\hat{\phi}$ & A smooth potential field that corrects for the boundary conditions \\
\hline$\Phi_{\mathrm{P}}$ & Applied electrical potential to the anode \\
\hline
\end{tabular}

\section{The initiation of filament growth}

We begin by summarising a recent model [18] for the initiation of filament growth before proceeding to extend it to the growth rate of filaments. Throughout the analysis we assume, in line with numerous previous investigations (e.g. [14-17]), that the electrolyte remains electroneutral and that the molar volume of $\mathrm{Li}^{+}$within the electrolyte vanishes, $\Omega_{\mathrm{e}}=0$. This second assumption is based on the notion that the Li lies within a rigid ceramic skeleton of the electrolyte that does not deform upon removal/addition of a $\mathrm{Li}$ atom. Throughout this manuscript we employ Cartesian tensor notation.

Consider a symmetric cell with Li metal electrodes and no external mechanical loading. A filament of initial length $a=a_{0}$ is idealised as a climbing dislocation with a Burgers vector of magnitude $b$; it emanates at a right angle from the Li cathode ${ }^{1}$ that is taken to be ground, see Fig. 1. We define the overpotential $\eta\left(x_{i}\right)$ of $\mathrm{Li}^{+}$in the electrolyte at location $x_{i}$ with respect to

\footnotetext{
${ }^{1}$ The cathode is defined as the electrode at which the reduction reaction occurs.
} 
the $\mathrm{Li}^{+}$in the cathode as $\eta \equiv-(\phi+\mathcal{U})$, where $\phi\left(x_{i}\right)$ is the electrical potential at location $x_{i}$ in the electrolyte and $\mathcal{U}$ is the open circuit potential (also known as the equilibrium potential) of $\mathrm{Li}^{+}$in the $\mathrm{Li}$ electrode with respect to the $\mathrm{Li}^{+}$in the electrolyte, see [18] for the definition of $\mathcal{U}$. The occupancy of the Li sites along the filament is such that the chemical potential of the $\mathrm{Li}^{+}$in the filament over the entire length of the filament equals that of $\mathrm{Li}^{+}$within the adjacent electrolyte. This is an immediate consequence of the ability of $\mathrm{Li}^{+}$to cross the interface rapidly and bring the $\mathrm{Li}^{+}$in the electrolyte and the $\mathrm{Li}^{+}$in the filament quickly into equilibrium with each other. The net flux of $\mathrm{Li}^{+}$across the filament flanks therefore vanishes. Growth of the filament, if it occurs, is by the fracture and wedging open of the electrolyte at the filament tip, as illustrated in Fig. 1. The fracture and wedging open of the electrolyte require energy, and this is provided by the loss of free-energy associated with the flux of $\mathrm{Li}^{+}$from the electrolyte into the filament, at constant chemical potential $\mu_{\text {tip }} \mathrm{Of} \mathrm{Li}^{+}$at the filament tip.

Consider the growth of the filament by a distance $\Delta a$ in an electrolyte of shear modulus $G$, Poisson's ratio $v$ and thickness $B$ in the $x_{3}$-direction (Fig. 1). Filament growth occurs at a constant chemical potential of $\mathrm{Li}^{+}$given by

$$
\mu_{\text {tip }}=\mu_{\mathrm{e}}^{0}+F \phi_{\text {tip }}+R T \ln \left(\frac{\theta_{\mathrm{e}}^{0}}{1-\theta_{\mathrm{e}}^{0}}\right)
$$

with $R$ the universal gas constant, $\mu_{\mathrm{e}}^{0}$ the reference chemical potential of $\mathrm{Li}^{+}$in the electrolyte, $\theta_{\mathrm{e}}^{0}$ the occupancy of $\mathrm{Li}^{+}$in the electrolyte, $\phi_{\text {tip }}$ the electrical potential of the electrolyte at the filament tip and $F$ the Faraday constant. Then, with $\eta_{\text {tip }} \equiv-\left(\phi_{\text {tip }}+\mathcal{U}\right)$ denoting the value of $\eta\left(x_{i}\right)$ at the filament tip, the free-energy release due to filament advance by $B \Delta a$ is [18] ${ }^{2}$

$$
\Delta \mathcal{L}_{\text {tip }}=\left\{2 \gamma_{\mathrm{e} / \mathrm{Li}}+\rho_{\mathrm{m}} b\left[F \eta_{\text {tip }}-T \frac{s_{\text {tip }}}{\theta_{\text {tip }}}+\left(\frac{1}{\theta_{\text {tip }}}-1\right) h_{\mathrm{v}}\right]+\frac{G b^{2}}{4 \pi(1-v) a_{0}}\right\} B \Delta a .
$$

Here, $\gamma_{\mathrm{e} / \mathrm{Li}}$ is the energy per unit area of the electrolyte/Li interface while $\rho_{\mathrm{m}}$ and $h_{\mathrm{v}}$ are the theoretical molar density of $\mathrm{Li}$ and enthalpy of formation of vacancies in $\mathrm{Li}$, respectively. The specific mixing entropy, $s_{\text {tip }}$, of $\mathrm{Li}$ within the filament at the filament tip is related to the occupancy $\theta_{\text {tip }}$ of the Li lattice sites within the filament at the tip via

$$
s_{\text {tip }} \equiv-R\left[\theta_{\text {tip }} \ln \theta_{\text {tip }}+\left(1-\theta_{\text {tip }}\right) \ln \left(1-\theta_{\text {tip }}\right)\right],
$$

where the value of $\theta_{\text {tip }}$ is set by the requirement that the chemical potential of $\mathrm{Li}^{+}$within the filament and adjacent electrolyte are equal at the tip. Consequently, $\theta_{\text {tip }}$ is given by

$$
\frac{1}{\theta_{\text {tip }}}=1+\exp \left[\frac{F \eta_{\text {tip }}-h_{\mathrm{v}}}{R T}\right] \text {. }
$$

Filament growth can occur if $\Delta \mathcal{L}_{\text {tip }} \leq 0$ (with $\Delta a>0$ ) and this gives the critical value of $\eta_{\text {tip }}$ required to initiate filament growth (labelled $\eta_{\mathrm{c}}$ ) as

$$
\eta_{\mathrm{c}}=\frac{1}{F}\left[T \frac{s_{\mathrm{tip}}}{\theta_{\mathrm{tip}}}-\left(\frac{1}{\theta_{\mathrm{tip}}}-1\right) h_{\mathrm{v}}\right]-\frac{1}{F \rho_{\mathrm{m}} b}\left(2 \gamma_{\mathrm{e} / \mathrm{Li}}+\frac{G b^{2}}{4 \pi(1-v) a_{0}}\right) .
$$

\footnotetext{
${ }^{2}$ There is a typographical error in our recent paper [18] where $s_{\text {tip }}$ should be replaced with $s_{\text {tip }} / \theta_{\text {tip }}$ in Eqs. (2.32) through (2.35).
} 
With $h_{\mathrm{v}}=50 \mathrm{~kJ} \mathrm{~mol}^{-1}$ [22], it follows that $h_{\mathrm{v}} /(R T) \gg 1$ at room temperature $T=300 \mathrm{~K}$. Therefore, at room temperature $\theta_{\text {tip }} \approx 1$ and $s_{\text {tip }} \rightarrow 0$ implying that, to a high degree of accuracy, Eq. (2.5) reduces to

$$
\eta_{\mathrm{c}} \approx-\frac{1}{F \rho_{\mathrm{m}} b}\left(2 \gamma_{\mathrm{e} / \mathrm{Li}}+\frac{G b^{2}}{4 \pi(1-v) a_{0}}\right) .
$$

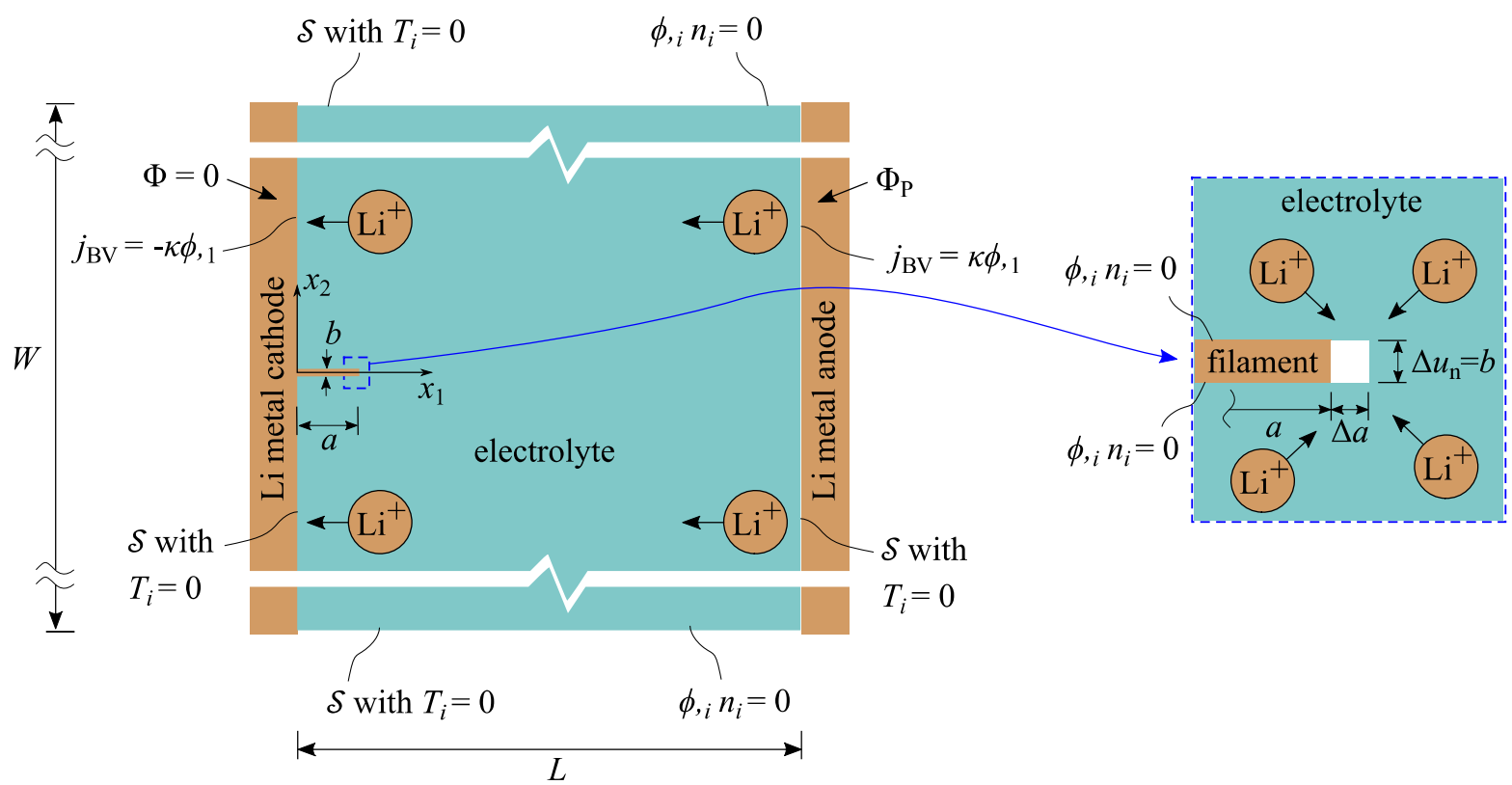

Figure 1: Sketch of a symmetric cell comprising a single ion-conductor ceramic electrolyte of thickness $L$ and width $W$ and Li electrodes covering the electrolyte. A filament of length $a$ (initial length $a_{0}$ ) and Burgers vector $b$ emanates at right angle from the cathode taken to be ground while the anode is maintained at an electrical potential $\Phi_{\mathrm{P}}$. The global co-ordinate system $\left(x_{1}, x_{2}\right)$ with its origin at the root of the filament is also indicated. The boundary conditions on the electrolyte are also indicated with the inset showing the extension of the filament by $\Delta a$ accompanied by fracture of the electrolyte, wedging open of the electrolyte by $b$ and the flux of $\mathrm{Li}^{+}$into the filament through its tip.

Experimental measurements on the short-circuiting of symmetric cells are usually presented in terms of a critical current density $i_{\mathrm{CCD}}$. In [18], this current density was interpreted as the current density required to initiate filament growth. The calculation of $i_{\mathrm{CCD}}$ requires us to relate the nominal cell current density, $j_{\infty}$, to the overpotential at the filament tip, $\eta_{\text {tip }}$, and therefore requires a solution of the electrochemical equations for $\mathrm{Li}^{+}$flux within the electrolyte. To do so, treat the electroneutral electrolyte as a linear dielectric with a conductivity $\kappa$ for the $\mathrm{Li}^{+}$ flux such that the $\mathrm{Li}^{+}$flux is $j_{i}=-\kappa \phi_{, i}$. Then, the electrochemical balance laws reduce to Laplace's equation $\phi_{, i i}=\eta_{, i i}=0$ with appropriate boundary conditions. These boundary conditions are: (i) zero flux of $\mathrm{Li}^{+}$across the filament flanks, and across the interface with freespace, as expressed by the Neumann boundary condition $\eta_{, i} n_{i}=0$, where $n_{i}$ is the unit outward normal along the respective interface and (ii) the Butler-Volmer boundary conditions along the electrode interfaces, as follows. The anode at $x_{1}=L$ is maintained at an electrical potential $\Phi_{\mathrm{P}}$ and the flux is related to this potential via 


$$
\left.\frac{\partial \eta}{\partial x_{1}}\right|_{x_{1}=L}=\frac{\bar{J}_{0}}{\kappa}\left\{\exp \left[\frac{-\beta F\left(\Phi_{\mathrm{P}}+\eta\right)}{R T}\right]-\exp \left[\frac{(1-\beta) F\left(\Phi_{\mathrm{P}}+\eta\right)}{R T}\right]\right\} .
$$

Likewise, the cathode along $x_{1}=0$ is taken to be ground, and the flux there is of the form

$$
\left.\frac{\partial \eta}{\partial x_{1}}\right|_{x_{1}=0}=-\frac{\bar{J}_{0}}{\kappa}\left\{\exp \left[\frac{-\beta F \eta}{R T}\right]-\exp \left[\frac{(1-\beta) F \eta}{R T}\right]\right\} \text {. }
$$

The Butler-Volmer symmetry factor $\beta$ in Eqs. (2.7) and (2.8) satisfies $0 \leq \beta \leq 1$ and the exchange current density $\bar{J}_{0}$ is related to the electrode/electrolyte interfacial resistance $Z$ via $\bar{J}_{0}=R T /(Z F)[18]$.

Consider the problem sketched in Fig. 1. The symmetric cell is loaded by a nominal current density $j_{\infty}$ (positive in value when the current is in the positive $x_{1}$-direction), and $b$ is much less than all leading dimensions of the electrolyte. The boundary value problem for a stationary filament (i.e. not growing since $\eta_{\text {tip }}<\eta_{\mathrm{c}}$ ) can be solved analytically to give

$$
\eta_{\text {tip }}=\frac{j_{\infty} a_{0}}{\kappa}+\frac{2 R T}{F} \sinh ^{-1}\left(\frac{j_{\infty} Z F}{2 R T}\right) \approx j_{\infty}\left(Z+\frac{a_{0}}{\kappa}\right),
$$

where we have taken $\beta=1 / 2$ and the approximation assumes $j_{\infty} Z F /(2 R T) \ll 1$. The minimum current $-j_{\infty}=i_{\min }$ to initiate filament growth in the electrolyte is obtained by equating $\eta_{\text {tip }}$ to $\eta_{\mathrm{c}}$. Now use the linearized form in (2.9), along with the approximate expression (2.6) for $\eta_{c}$, to obtain

$$
i_{\min } \approx \frac{1}{F \rho_{\mathrm{m}}\left(Z+\frac{a_{0}}{\kappa}\right)}\left[\frac{2 \gamma_{\mathrm{e} / \mathrm{Li}}}{b}+\frac{G b}{4 \pi(1-v) a_{0}}\right] .
$$

We observe from (2.10) that there exists a critical value of $b$, labelled $b_{\mathrm{CCD}}$, for which $i_{\min }$ is minimised. This critical value of Burgers vector magnitude is given by

$$
b_{\mathrm{CCD}} \approx \sqrt{\frac{8 \pi(1-v) a_{0} \gamma_{\mathrm{e} / \mathrm{Li}}}{G}},
$$

and the value of $i_{\text {min }}$ corresponding to $b_{\mathrm{CCD}}$ is labelled the critical current density $i_{\mathrm{CCD}}$. It is assumed implicitly that, for a given value of $a_{0}$, there exist filaments with a spectrum of values for $b$ within the electrolyte: the filament that grows at the lowest current has $b=b_{\mathrm{CCD}}$ and this sets the critical current density.

This brief summary of the model presented in [18] for the initiation of filament growth serves as the starting point for addressing the main challenge of this paper: at what rate will filaments grow when a current density $\left|j_{\infty}\right| \geq i_{\mathrm{CCD}}$ is imposed on the symmetric cell?

\section{A model for the growth rate of Li filaments}

Here, we present the analysis for predicting the growth rate of a filament emanating at a right angle from the cathodic interface in a symmetric cell with an imposed anodic potential $\Phi_{\mathrm{P}}$ and the cathode taken to be ground (i.e. a symmetric cell with an imposed voltage rather than an imposed current). The cell, with an electrolyte of dimension $L \times W$ (Fig. 1), is not subjected to external mechanical loading and Maxwell stresses due to charges at the surface of the 
electrolyte are neglected ${ }^{3}$. However, we emphasize that the approach can be readily generalised for an arbitrary cell geometry and any imposed electrical/mechanical loading.

Consider a filament with Burgers vector of magnitude $b$ and current length $a$ growing at a rate $v \equiv \dot{a}$ (Fig. 1). The rate of free-energy release due to a filament growth rate $v$ follows from (2.2) as

$$
\dot{\mathcal{L}}_{\text {tip }}=B v\left\{2 \gamma_{\mathrm{e} / \mathrm{Li}}+\rho_{\mathrm{m}} b\left[F \eta_{\text {tip }}-T \frac{s_{\text {tip }}}{\theta_{\text {tip }}}+\left(\frac{1}{\theta_{\text {tip }}}-1\right) h_{\mathrm{v}}\right]+\frac{G b^{2}}{4 \pi(1-v) a}\right\} .
$$

Recall that the critical filament tip overpotential for which $\dot{\mathcal{L}}_{\text {tip }}=0$ is given by (2.5), with $a_{0}$ replaced by $a$. It is convenient to re-write (3.1) succinctly as

$$
\dot{\mathcal{L}}_{\text {tip }}=B v F \rho_{\mathrm{m}} b\left(\eta_{\text {tip }}-\eta_{\mathrm{c}}\right) \text {, }
$$

which gives the rate of loss of free-energy at the tip of the growing filament. This loss of freeenergy is dissipated at the filament tip and, motivated by (3.2), we propose a phenomenological relation for the tip dissipation rate as

$$
\dot{\mathcal{D}}=B \frac{\left(\eta_{\mathrm{c}}-\eta_{\text {tip }}\right)^{2}}{R_{\text {tip }}},
$$

where $R_{\mathrm{tip}} / B$ is the resistance that $\mathrm{Li}^{+}$encounters for its flux through the filament tip. The velocity of filament growth follows by equating the rate of loss of free-energy $-\dot{\mathcal{L}}_{\text {tip }}$ to the dissipation $\dot{\mathcal{D}}$ at the filament tip, giving

$$
v=\frac{1}{F \rho_{\mathrm{m}} b} \frac{\left(\eta_{\mathrm{c}}-\eta_{\mathrm{tip}}\right)}{R_{\mathrm{tip}}}
$$

The $\mathrm{Li}^{+}$flux per unit thickness, $I_{\text {tip }}$, into the filament tip required to grow the filament at this rate is then given by mass conservation as

$$
I_{\text {tip }}=-F \rho_{\mathrm{m}} b \theta_{\text {tip }} v=-\frac{\theta_{\text {tip }}\left(\eta_{\mathrm{c}}-\eta_{\text {tip }}\right)}{R_{\text {tip }}} \approx-\frac{\left(\eta_{\mathrm{c}}-\eta_{\text {tip }}\right)}{R_{\text {tip }}},
$$

where the minus sign denotes that flux into the tip is defined as negative and the approximation uses the fact that $\theta_{\text {tip }} \approx 1$. It now remains to calculate $\eta_{\text {tip }}$ for the growing filament. Now, the spatial distribution of the overpotential, $\eta$, in the electrolyte remains unaffected by the presence of a stationary filament and is given trivially by (2.9). The problem is more complicated for the growing filament as the filament tip serves as a sink for $\mathrm{Li}^{+}$, with the flux of $\mathrm{Li}^{+}$into the filament tip influencing the overall spatial distribution of $\eta$. The sink also creates computational difficulties in terms of a direct numerical solution and here we propose a superposition method to solve for $\eta$.

Consider a filament with a Burgers vector of magnitude $b$ and a polar co-ordinate system with the filament tip located at the origin $r=0$. In 2D, the electric potential at a radius $r$ from the

\footnotetext{
${ }^{3}$ Typical electric fields rarely exceed $1000 \mathrm{Vm}^{-1}(1 \mathrm{~V}$ applied over an electrolyte of thickness $1 \mathrm{~mm})$. Given that the relative permittivity of LLZO $\approx 50$ [23], the Maxwell stresses associated with this electric field are on the order of $10^{-4} \mathrm{~Pa}$ and are hence negligible compared to the stresses generated by the filament, which can be in the GPa range near the filament tip.
} 
filament tip, due to a line sink (along the $x_{3}$-direction in Fig. 1) of strength $\mathcal{C}$ in an infinite medium is

$$
\tilde{\phi}(r)=\mathcal{C} \ln (r / b) .
$$

Here, we have normalised (3.6) such that $\tilde{\phi}=0$ at $r=b$ which is taken to be the filament tip, i.e. $\tilde{\phi}_{\text {tip }}=0$. The potential field (3.6) satisfies the electroneutral Gauss's law $\tilde{\phi}_{, i i}=0$ for $r>$ 0 , but also results in a current flux into the filament tip. The current flux $\tilde{I}_{\text {tip }}$ per unit thickness of the electrolyte into the tip associated with this potential field is readily calculated by considering a circular contour $\Gamma$ centred at $r=0$. The flux across unit length of this contour is $\tilde{\jmath}_{i} \tilde{n}_{i}$, where $\tilde{n}_{i}$ is the unit outward normal to the contour and $\tilde{\jmath}_{i}=-\kappa \tilde{\phi}_{, i}$. Since $\tilde{\phi}_{, i i}=0$ for $r>$ 0 , this flux represents the flux into the sink located at $r=0$ and follows as

$$
\tilde{I}_{\text {tip }}=\int_{\Gamma} \tilde{J}_{i} \tilde{n}_{i} d \Gamma=-\kappa \int_{\Gamma} \frac{\partial \tilde{\phi}}{\partial r} d \Gamma=-2 \pi \kappa \mathcal{C} .
$$

Note that the field $\tilde{\phi}$ gives a current flux into the filament tip, but it does not satisfy the boundary conditions imposed on the symmetric cell; imposition of these boundary conditions along with (3.5) will set the value of $\tilde{I}_{\text {tip }}$ via the value obtained for $\mathcal{C}$.

In order to satisfy the required boundary conditions, we recall linearity of Gauss's law and write the potential within the electrolyte as $\phi=\widehat{\phi}+\tilde{\phi}$ such that the overpotential within the electrolyte with respect to the cathode is $\eta \equiv-(\phi+\mathcal{U})=\hat{\eta}-\tilde{\phi}$, where $\hat{\eta}=-(\hat{\phi}+\mathcal{U})$ and $\hat{\phi}$ is a smooth field that corrects for the boundary conditions. Recalling that $\widetilde{\phi}_{, i i}=0$, the solution of the total potential field reduces to solving $\hat{\eta}_{, i i}=0$ with the appropriate boundary conditions. These boundary conditions are:

(i) The Neumann boundary conditions that enforce zero flux across the boundaries with free-space, viz. $\hat{\eta}_{, 2}=\tilde{\phi}_{, 2}$ along $x_{2}= \pm W / 2$.

(ii) Similarly, zero-flux conditions are imposed along the filament flanks such that $\hat{\eta}_{, 2}=0$, where we have used the fact that $\widetilde{\phi}_{, 2}=0$ along the filament flanks.

(iii) Finally, we impose the Robin-like boundary conditions for the Butler-Volmer flux relations along the electrode/electrolyte interfaces. These are

$$
\hat{\eta}_{, 1}=\frac{R T}{F Z \kappa}\left\{\exp \left[\frac{-\beta F\left(\Phi_{\mathrm{P}}+\hat{\eta}-\tilde{\phi}\right)}{R T}\right]-\exp \left[\frac{(1-\beta) F\left(\Phi_{\mathrm{P}}+\hat{\eta}-\tilde{\phi}\right)}{R T}\right]\right\}+\tilde{\phi}_{, 1},
$$

along the anode interface located at $x_{1}=L$ and

$$
\hat{\eta}_{, 1}=-\frac{R T}{F Z \kappa}\left\{\exp \left[\frac{-\beta F(\hat{\eta}-\tilde{\phi})}{R T}\right]-\exp \left[\frac{(1-\beta) F(\hat{\eta}-\tilde{\phi})}{R T}\right]\right\}+\tilde{\phi}_{, 1},
$$

along the cathode interface located at $x_{1}=0$. The numerical solution of this boundary value problem for a given applied $\Phi_{\mathrm{P}}$ furnishes the distribution $\hat{\eta}\left(x_{1}, x_{2}\right)$ within the electrolyte for an assumed value of $\mathcal{C}$ and the filament tip overpotential follows as $\eta_{\text {tip }}=\hat{\eta}_{\text {tip }}(\mathcal{C})=\hat{\eta}\left(x_{1}=\right.$ $\left.a, x_{2}=0\right)$, since $\tilde{\phi}_{\text {tip }}=0$.

It remains to determine $\mathcal{C}$ using (3.5). The total current into the filament tip is $I_{\text {tip }}=\hat{I}_{\text {tip }}+\tilde{I}_{\text {tip }}$, where $\hat{I}_{\text {tip }}$ is the current due to the $\hat{\phi}$ field. Considering a circular contour centred at the filament 
tip and using the divergence theorem, it follows that $\hat{I}_{\text {tip }}=0$ since $\hat{\phi}$ is a smooth field that satisfies $\hat{\phi}_{, i i}=0$. Upon substituting (3.7) into (3.5), we obtain

$$
\mathcal{C}=\frac{\eta_{\mathrm{c}}-\hat{\eta}_{\mathrm{tip}}(\mathcal{C})}{2 \pi \kappa R_{\mathrm{tip}}}
$$

giving an implicit relation for $\mathcal{C}$. We solve (3.10) iteratively for $\mathcal{C}$ and the velocity of the filament at a given length $a$ follows from (3.5) and (3.7) as

$$
v(a)=\frac{2 \pi \kappa \mathcal{C}}{F \rho_{\mathrm{m}} b} .
$$

This procedure needs to be repeated at discrete values of $a$ to obtain the relation $\dot{a}=v(a)$ and thereby the evolution of the filament length $a$ with time $t$. The numerical solution of Laplace's equation for the overpotential $\hat{\eta}$ was performed using the commercial finite element (FE) package ABAQUS with Matlab used to solve (3.10) iteratively. Abaqus2Matlab [25] was employed to connect ABAQUS and Matlab.

The growing filament influences the magnitude of the current density through the cell for a fixed applied anodic potential $\Phi_{\mathrm{P}}$ and it is worth clarifying this issue. To help define the problem and connect with the results for initiation of growth in [18], we define a current density $j_{\infty}$ that ensues in the symmetric cell due to application of an anodic voltage $\Phi_{\mathrm{P}}$ in the absence of a growing filament. With $\beta=1 / 2, \Phi_{\mathrm{P}}$ and $j_{\infty}$ are related via

$$
\Phi_{\mathrm{P}}=-\left[\frac{j_{\infty} L}{\kappa}+\frac{4 R T}{F} \sinh ^{-1}\left(\frac{Z F j_{\infty}}{2 R T}\right)\right]
$$

which reduces to the linearized expression

$$
j_{\infty} \approx-\frac{\Phi_{\mathrm{P}}}{(L / \kappa+2 Z)}
$$

for $Z F j_{\infty} /(2 R T) \ll 1$. For the case of a growing filament, we can define three currents (per unit depth in the $x_{3}$-direction): (i) an anodic ionic current $J_{\mathrm{A}}$ quantifying the flux of $\mathrm{Li}^{+}$ions across the anodic interface, neglecting the contribution from the filament tip; (ii) a cathodic ionic current $J_{C}$ quantifying the flux of $\mathrm{Li}^{+}$ions across the cathodic interface and (iii) an electron current $J_{\mathrm{el}}$ in the external circuit which is typically the current measured in experiments. For the case of an isolated growing filament emanating from the cathodic interface as sketched in Fig. 1, conservation of $\mathrm{Li}^{+}$ions requires $J_{\mathrm{A}}=J_{\mathrm{C}}+I_{\text {tip }}$ and the electron current $J_{\mathrm{el}}=J_{\mathrm{A}}$ to ensure overall charge neutrality of the cell. The divergence between the anodic and cathodic currents increases with increasing growth rate of the filament and we shall demonstrate this via the average electrode current densities $j_{\mathrm{A}} \equiv J_{\mathrm{A}} / W$ and $j_{\mathrm{C}} \equiv J_{\mathrm{C}} / W$ that are calculated as

$$
j_{\mathrm{A}}=\frac{1}{W} \int_{-W / 2}^{W / 2} j_{1}\left(x_{1}=L, x_{2}\right) d x_{2}
$$

and

$$
j_{\mathrm{C}}=\frac{1}{W} \int_{-W / 2}^{W / 2} j_{1}\left(x_{1}=0, x_{2}\right) d x_{2}
$$

at the anodic and cathodic interfaces, respectively. We emphasize that such an integration is needed as filament growth results in the electric fields within the electrolyte no longer 
remaining one-dimensional: the flux $j_{1}$ is spatially non-uniform over the electrode/electrolyte interfaces.

The above formulation is written specifically for growth of the filaments from the cathode (or plating electrode). As observed recently [13], these filaments shrink when the current direction is reversed and the electrode from which the filament emerges switches to becoming the anode (or stripping electrode). In Appendix A, we present the analysis for this case.

\section{Predictions for the propagation rate of a filament}

Numerical predictions are presented for the rate of growth of the isolated filament of initial length $a_{0}$ as sketched in Fig. 1. The electrolyte is taken to be LLZO and all required material parameters have either been directly experimentally measured or computed from density functional theory (DFT) calculations. The $\mathrm{Li}^{+}$conductivity $\kappa=0.46 \mathrm{mS} \mathrm{cm}^{-1}$ [9], shear modulus $G=60 \mathrm{GPa}$ and Poisson's ratio $v=0.2$ [24] are measured values. On the other hand, the surface energies are available from DFT calculations [8]: the surface energies of LLZO and Li are $\gamma_{\mathrm{LLZO}}=0.84 \mathrm{~J} \mathrm{~m}^{-2}$ and $\gamma_{\mathrm{Li}}=0.45 \mathrm{~J} \mathrm{~m}^{-2}$, respectively, while the work of adhesion between LLZO and Li is $W_{\text {adh }}=0.67 \mathrm{~J} \mathrm{~m}^{-2}$. The energy of the LLZO/Li interface is then given by the Born-Haber cycle as $\gamma_{\mathrm{e} / \mathrm{Li}} \equiv \gamma_{\mathrm{LLZO} / \mathrm{Li}}=\gamma_{\mathrm{LLZO}}+\gamma_{\mathrm{Li}}-W_{\mathrm{adh}}=0.62 \mathrm{~J} \mathrm{~m}^{-2}$. The theoretical molar density of $\mathrm{Li}$ is $\rho_{\mathrm{m}}=76286 \mathrm{~mol} \mathrm{~m}^{-3}\left(\rho_{\mathrm{m}} \equiv \rho_{\mathrm{Li}} / M_{\mathrm{Li}}\right.$ where the density of Li $\rho_{\mathrm{Li}}=534 \mathrm{~kg} \mathrm{~m}^{-3}$ and molar mass $M_{\mathrm{Li}}=7 \mathrm{~g} \mathrm{~mol}^{-1}$ ) and the enthalpy of vacancy formation in $\mathrm{Li}$ is $h_{\mathrm{v}}=50 \mathrm{~kJ} \mathrm{~mol}^{-1}$ [22]. The flux of $\mathrm{Li}^{+}$across the electrolyte/electrode interfaces is characterised in terms of the resistance $Z$ and the Butler-Volmer symmetry factor $\beta$. Here, we present predictions for $Z=5 \Omega \mathrm{cm}^{2}$ that is representative of the high-quality interfaces investigated in $[8,9]$ and set $\beta=1 / 2$. We emphasize that there are no direct measurements of $\beta$. However, the applied voltages are sufficiently low that the linearized versions of the ButlerVolmer flux laws (2.7) and (2.8) are valid to a very high degree of accuracy: in this linearized limit $\beta$ plays no role. Consequently, the value of $\beta$ has very little influence on the results. Finally, we emphasise that the governing equations for the symmetric cell can be directly written in terms of the overpotential $\eta$ : direct knowledge of the open circuit potential $\mathcal{U}$ is not required for relating the filament growth rates to the imposed nominal cell currents. Thus, for the purpose of this analysis, we do not need to specify a value for $\mathcal{U}$. All results are presented at a temperature $T=300 \mathrm{~K}$ and for an electrolyte of thickness $L=1000 \mu \mathrm{m}$ and width $W=$ $10 L$, in line with a large number of experiments [6-11].

The simulations were conducted at a constant applied anodic potential $\Phi_{\mathrm{P}}$ with the cathode taken to be ground. Predictions of the critical current density $i_{\mathrm{CCD}}$ from [18] obtained by substituting (2.11) into (2.10) are shown in Fig. 2 (the $\ell=0$ case): $i_{\text {CCD }}$ decreases with increasing $a_{0}$ both due to an increase in the overpotential $\left|\eta_{\text {tip }}\right|$ at the filament tip and the fact that the elastic energy required to wedge open the ceramic electrolyte decreases with increasing $a_{0}$. Now, limit the consideration to the choice of $a_{0}=5 \mu \mathrm{m}$ and focus on the influence of the non-dimensional filament tip resistance $\kappa R_{\text {tip }}$ in setting the growth rate of the filament. Loading is applied via a fixed anodic voltage $\Phi_{\mathrm{P}}$ in order to make for easy comparison with 
the initiation results reported in [18] and also connect with experiments; however, we shall quote results in terms of the current density $j_{\infty}$, as defined by the current density in the absence of filament growth for the given $\Phi_{\mathrm{P}}$.

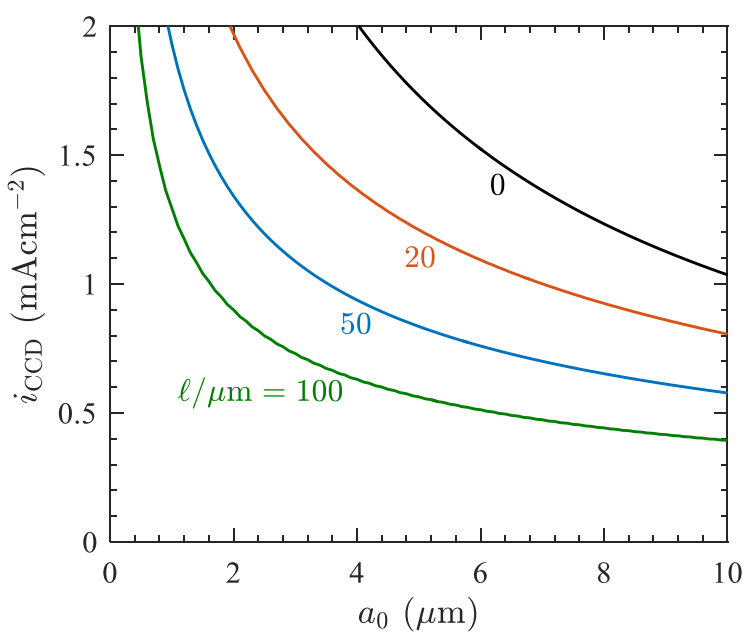

Figure 2: Predictions of the critical current density $i_{\mathrm{CCD}}$ as a function of the initial filament length $a_{0}$ for a cell with interfacial resistance $Z=5 \Omega \mathrm{cm}^{2}$ and selected values of the void size $\ell$. Reproduced from [18].

Two cases are considered: (i) ideal electrical contact between the cathode and electrolyte, i.e. $Z$ is spatially uniform over the interface and (ii) the situation where a void of size $\ell$ forms on the cathodic interface such that contact is lost between the electrolyte and cathode over this region and hence $Z=\infty$ in that zone (Fig. 3). In both cases, $Z$ is spatially uniform over the anode/electrolyte interface where there is contact.

a

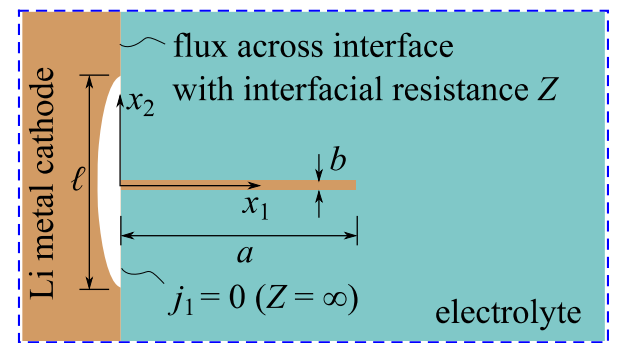

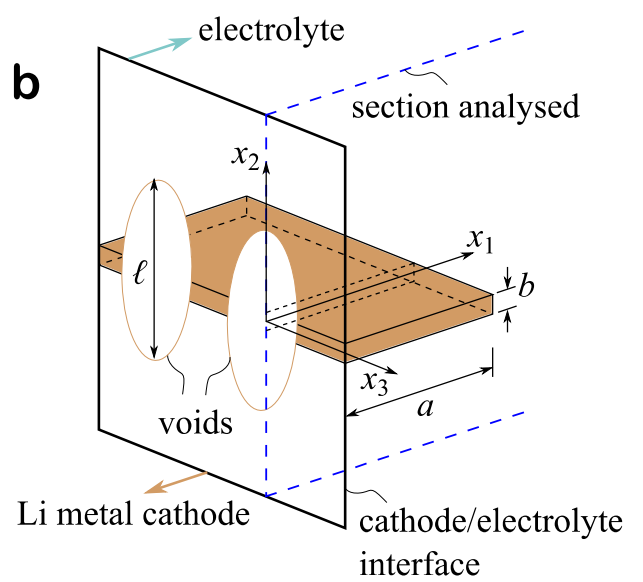

Figure 3: (a) Sketch of the 2D problem of a filament of length $a$ emanating at a right angle from the centre of a void of size $\ell$ in the electrode along the electrolyte interface. The co-ordinate system $\left(x_{1}, x_{2}\right)$ is marked and the void is modelled as a patch of size $\ell$ with infinite ionic resistance $(Z=\infty)$. (b) A 3D sketch of the void along the interface showing the electrical connection of the filament to the electrode. The plane indicating the section analysed in the $2 \mathrm{D}$ problem is also marked. 


\subsection{Reference case of local equilibrium at the filament tip}

Here, we restrict attention to the choice $R_{\text {tip }}=0$. In this case, local equilibrium is maintained at the filament tip with filament growth occurring such that $\dot{\mathcal{L}}_{\text {tip }}=0$ and Eq. (3.10) requiring that $\eta_{\text {tip }}=\eta_{\mathrm{c}}$. First, consider the case of ideal contact between cathode and electrolyte (no void with $\ell=0$ ). When a cell current $\left|j_{\infty}\right| \geq i_{\mathrm{CCD}}$ is applied, the filament with a Burgers vector $b=$ $b_{\mathrm{CCD}}$ will grow at a rate $v$ such that $\eta_{\text {tip }}=\eta_{\mathrm{c}}$ throughout the growth process. Predictions of the temporal evolution of the filament length $a$ with time $t$ are included in Fig. $4 \mathrm{a}$ with time $t=0$ the instant when electric loading of the cell was initiated and the filament length $a=a_{0}$. Results are shown for two values of $\left|j_{\infty}\right|=i_{\text {CCD }}=1.73 \mathrm{~mA} \mathrm{~cm}^{-2}$ and $\left|j_{\infty}\right|=2 \mathrm{~mA} \mathrm{~cm}^{-2}$. In both cases, $b=b_{\mathrm{CCD}}=32.4 \mathrm{~nm}$ as specified by Eq. (2.11). The growth rate $\dot{a}$ increases with increasing time (or equivalently filament length $a$ ). This is because the equilibrium filament tip overpotential $\left|\eta_{\mathrm{c}}\right|$ given by (2.5) (with $a_{0}$ replaced by $a$ ) decreases with increasing $a$ and thus a higher growth rate is required to reduce $\eta_{\text {tip }}$ and maintain equilibrium at the filament tip. The growth rates increase with increasing $\left|j_{\infty}\right|$ as a higher current provides a larger driving force, $\eta_{\text {tip }}$, for filament growth. It is interesting to note that even at $\left|j_{\infty}\right|=i_{\mathrm{CCD}}$ we have a welldefined $a$ versus $t$ response even though $\dot{a}=0$ at $t=0$. This is because to a reasonable approximation for the $\left|j_{\infty}\right|=i_{\mathrm{CCD}}, \dot{a} \propto \sqrt{a-a_{0}}$ and thus $\left(a-a_{0}\right) \propto t^{2}$.
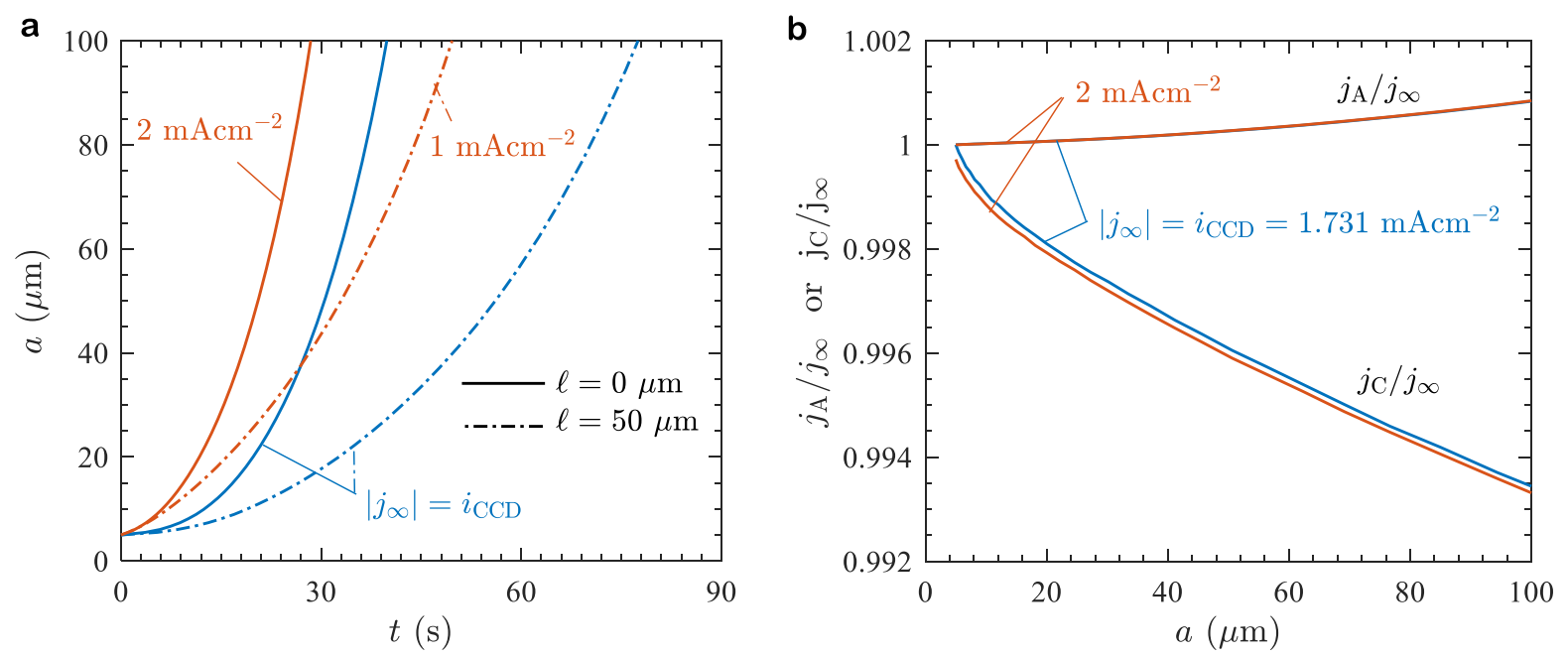

Figure 4: (a) Predictions of the evolution of the filament length $a$ with time $t$ for a tip resistance $R_{\text {tip }}=$ 0 and a cathodic interface with no void $(\ell=0)$ and with a void having $\ell=50 \mu \mathrm{m}$. Results are shown in each case for two values of the imposed current: $\left|j_{\infty}\right|=i_{\mathrm{CCD}}$ and a higher value. Time $t=0$ corresponds to the instant that the current was imposed. (b) The predictions of the normalised anodic and cathodic ionic currents $j_{\mathrm{A}} / j_{\infty}$ and $j_{\mathrm{C}} / j_{\infty}$, respectively, as a function of filament length $a$ for the two imposed currents in (a) for the no void $(\ell=0)$ case. 
a

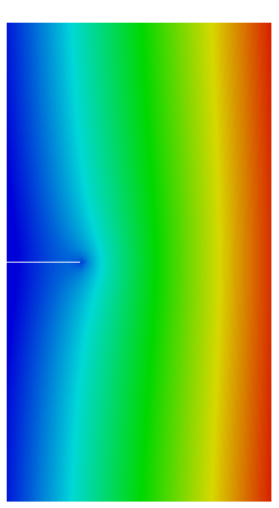

b

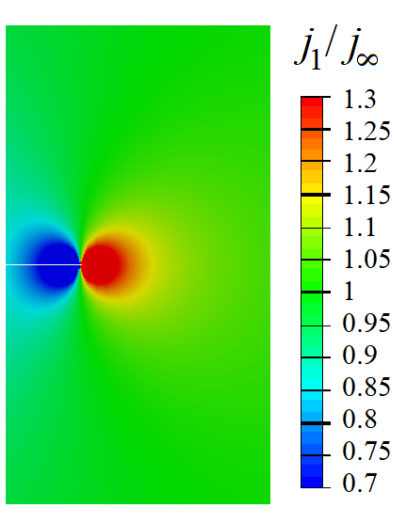

C
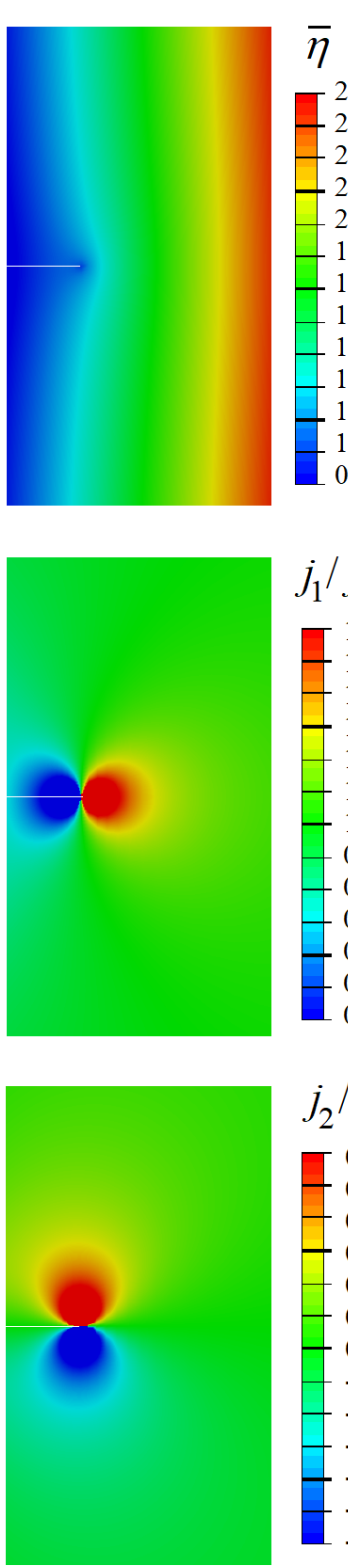

$\begin{array}{ll} & 2.7 \\ - & 2.55 \\ - & 2.4\end{array}$

2.5
-2.25

2.25
-1.95

1.8
-1.65

1.5
1.35

1.2
1.05

1.05

$j_{2} / j_{\infty}$

- 0.3

0.2
-0.15
-0.1

- 0.1

$-0.05$

-0.05
-0.1
-0.15

$-0.2$

$-0.3$ e

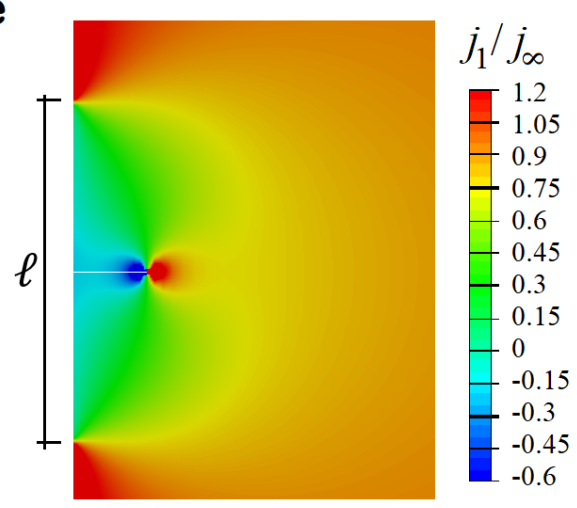

f

0
-0.05
-0.1

$-0.15$

$-0.25$

$10 \mu \mathrm{m}$ d $\bar{\eta}$

$\boldsymbol{E}^{3.7}$

$-3.4$

$-3.25$

3.1
-
-

2.95
-2.8

2.65

$-2.5$

$-2.35$

2.2

2.05

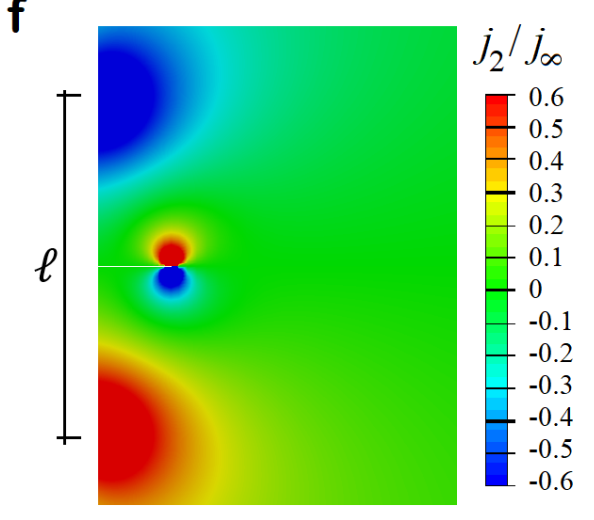

Figure 5: Predictions of the spatial distributions of the normalised (a,d) overpotential $\bar{\eta}$; (b,e) flux $j_{1} / j_{\infty}$ and (c,f) flux $j_{2} / j_{\infty}$ in the electrolyte after an initial filament of length $a_{0}=5 \mu \mathrm{m}$ has grown to $11 \mu \mathrm{m}$ with the filament tip resistance $R_{\text {tip }}=0$. The left column shows results for the case with no void $(\ell=$ 0 ), while the results in the right column are for a cathodic interface with $\ell=50 \mu \mathrm{m}$. In both cases the imposed current $\left|j_{\infty}\right|=i_{\mathrm{CCD}}$.

The spatial distribution of the normalised overpotential $\bar{\eta} \equiv-(\phi+\mathcal{U}) /\left(j_{\infty} Z\right)$ in the case of a non-growing (stationary) filament is trivially given by the one-dimensional field

$$
\bar{\eta}=\left(1+\frac{x_{1}}{\kappa Z}\right)
$$

with the co-ordinate $x_{1}=0$ at the cathode/electrolyte interface (Fig. 1). This distribution is significantly affected by the flux of $\mathrm{Li}^{+}$into the tip of the growing filament. Predictions of $\bar{\eta}$ are included in Fig. 5a for a nominal imposed current $\left|j_{\infty}\right|=i_{\mathrm{CCD}}$ at time $t=6 \mathrm{~s}$ when the 
filament has grown from $a_{0}=5 \mu \mathrm{m}$ to a length $a=11 \mu \mathrm{m}$. The tip of the growing filament locally reduces $\bar{\eta}$, as seen by curving of the $\bar{\eta}$ contours. The corresponding predictions of the normalised fluxes $j_{1} / j_{\infty}$ and $j_{2} / j_{\infty}$ are included in Figs. $5 \mathrm{~b}$ and $5 \mathrm{c}$, respectively. The growing filament results in an enhanced flux $j_{1} / j_{\infty}$ towards the tip from the anode side but the sink of current into the tip implies that the net current flowing into the cathode around the root of the filament is reduced, as seen in Fig. 5b. The effect of filament growth on $j_{2} / j_{\infty}$ is smaller, with concentration of a flux $j_{2} / j_{\infty}$ into the filament tip (recall $j_{2}=0$ throughout the electrolyte for the stationary filament).

The associated ionic currents $j_{\mathrm{A}} / j_{\infty}$ and $j_{\mathrm{C}} / j_{\infty}$ are plotted as a function of filament length $a$ in Fig. $4 \mathrm{~b}$ for both values of $\left|j_{\infty}\right|$ shown in Fig. 4a. First consider the case of $\left|j_{\infty}\right|=i_{\mathrm{CCD}}$. The ionic electrode currents are equal in this $\left|j_{\infty}\right|=i_{\mathrm{CCD}}$ case at $a=a_{0}$ since the filament velocity $v=0$ at filament length $a=a_{0}$. However, subsequently with increasing filament velocity, $j_{\mathrm{C}} / j_{\infty}$ drops with respect to $j_{\mathrm{A}} / j_{\infty}$ with the difference equal to the flux of $\mathrm{Li}^{+}$into the tip of the growing filament. Recall that the currents measured in experiments are electron currents in the external circuit. The electron current is equal in magnitude to the anodic current and we observe that for a fixed applied anodic potential, the current $j_{\mathrm{A}} / j_{\infty}$ increases slightly as the filament grows. This implies that the effective resistance of the cell decreases with filament growth, although this decrease is relatively minor prior to the filament penetrating across the remaining ligament of the electrolyte and short-circuiting the cell. The predictions for the higher value of $\left|j_{\infty}\right|$ included in Fig. $4 \mathrm{~b}$ reveal that $j_{\mathrm{A}} / j_{\infty}$ exceeds $j_{\mathrm{C}} / j_{\infty}$ even at $a=a_{0}$ : the filament immediately begins to grow with a finite velocity, since $\left|j_{\infty}\right|>i_{\mathrm{CCD}}$.

Now consider the case of a void (Fig. 3) in the Li electrode along the electrolyte/cathode interface. Recent observations [19] have suggested that voids of size $\ell \approx 20-100 \mu \mathrm{m}$ form during the consecutive plating and stripping phases of loading of the symmetric cell. In our recent paper [18], we demonstrate that such voids decrease $i_{\mathrm{CCD}}$ by increasing the overpotential $|\eta|$ at the cathodic interface in the vicinity of the void. Here, we analyse the effect of such voids on the growth rate of the filaments. In the two-dimensional (2D) approximation used here, we model the void of size $\ell$ on the cathodic interface as a patch of infinite ionic resistance $(Z=$ $\infty$ ) as shown in Fig. 3a, such that a zero-flux boundary is imposed in that region, with the usual Butler-Volmer boundary conditions imposed on the remainder of the interface. A filament of initial length $a_{0}$ is assumed to emanate from the centre of the void, as sketched in Fig. 3a. For this filament to grow by the deposition of $\mathrm{Li}^{+}$at its tip, it needs to be electrically connected to the cathode so as to acquire electrons and neutralize the $\mathrm{Li}^{+}$. We envisage that this is possible in the full three-dimensional (3D) situation: although the filament resembles an edge dislocation within an inserted sheet of $\mathrm{Li}$ in the $x_{1}-x_{3}$ plane, discrete voids are distributed along the root of the filament as sketched in Fig. 3b. Thus, the Li within the filament is electrically connected to the cathode and the $2 \mathrm{D}$ analysis here is of a section as indicated in Fig. 3b.

In order to place the filament growth rate results in context, we reproduce from [18] the predictions of $i_{\mathrm{CCD}}$ as a function of $a_{0}$ for different void sizes in Fig. 2. For any given initial 
filament length $a_{0},\left|\eta_{\text {tip }}\right|$ is increased by the presence of the void and consequently $i_{\mathrm{CCD}}$ decreases with increasing $\ell$. For example, as shown in Fig. $2, i_{\mathrm{CCD}}$ reduces from $i_{\mathrm{CCD}}=$ $1.73 \mathrm{~mA} \mathrm{~cm}^{-2}$ when no void was present to $0.83 \mathrm{~mA} \mathrm{~cm}^{-2}$ when a void of size $\ell=50 \mu \mathrm{m}$ is present. The growth of a filament of $b=b_{\mathrm{CCD}}=32.4 \mathrm{~nm}$ in the presence of a void of size $\ell=50 \mu \mathrm{m}$ is now considered. The $a$ versus $t$ responses are included in Fig. 4a for a cell subjected to a current $\left|j_{\infty}\right|=i_{\mathrm{CCD}}=0.83 \mathrm{~mA} \mathrm{~cm}^{-2}$ and to a higher current density of $\left|j_{\infty}\right|=$ $1.0 \mathrm{~mA} \mathrm{~cm}^{-2}$. In general, the behaviour is similar to the $\ell=0$ case with one key difference. While filament growth initiates at a lower current, the filament growth rates at $i_{\mathrm{CCD}}$ are higher in the cell with no void compared to the case with a void. This is because filament growth rates scale with the magnitude of the current rather than the amount by which the imposed current exceeds $i_{\mathrm{CCD}}$. The corresponding distributions of $\bar{\eta}, j_{1} / j_{\infty}$ and $j_{2} / j_{\infty}$ within the electrolyte when the filament has grown to $a=11 \mu \mathrm{m}$ are plotted in Figs. 5d through 5f, respectively, for the choice $\left|j_{\infty}\right|=i_{\mathrm{CCD}}=0.83 \mathrm{~mA} \mathrm{~cm}^{-2}$. Key differences are observed with respect to the no void case. The $\mathrm{Li}^{+}$sink has a more dramatic effect on $j_{1} / j_{\infty}$ when a void exists such that not only is $j_{1} / j_{\infty}$ enhanced for $x_{1}>a$ around the filament, i.e. increasing the flux towards the tip from the anode, but also $j_{1} / j_{\infty}$ reverses direction for $x_{1}<a$ around the filament with $\mathrm{Li}^{+}$ flowing from the cathode towards the filament tip (Fig. 5e). The effect of filament growth on $j_{2} / j_{\infty}$ (Fig. 5f) is less significant and, similar to the no void case, a small region of localised flux forms around the filament tip. Symmetry of the problem dictates that $j_{2}=0$ on the $x_{2}=$ 0 plane and so there is no direct flux of $\mathrm{Li}^{+}$into the filament tip in the $x_{2}$-direction. Although the Li within the filament is electrically connected to the cathode and is thereby maintained at $\phi=0$, there is no flux of $\mathrm{Li}^{+}$across the filament flanks since the chemical potential of the $\mathrm{Li}^{+}$ at any location along the flank equals that of the adjacent electrolyte.
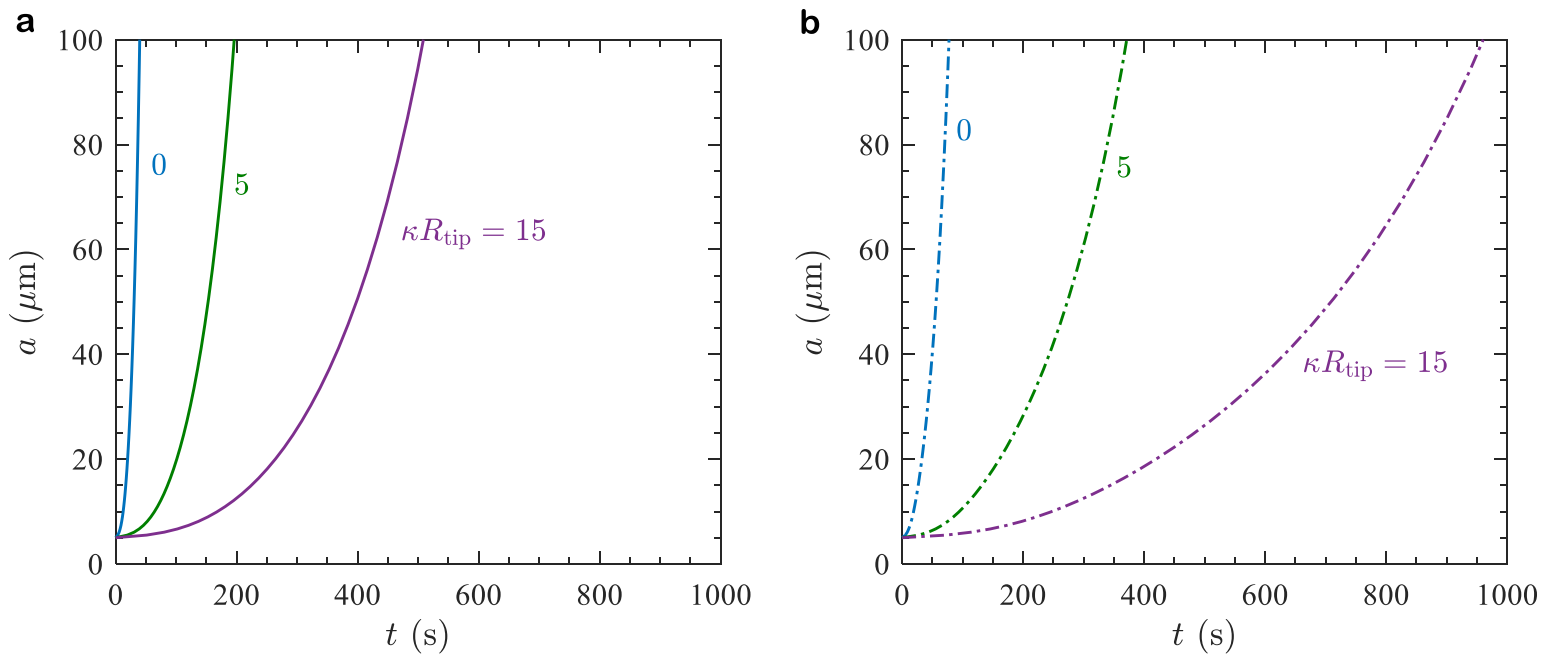

Figure 6: Predictions of the evolution of the filament length $a$ with time $t$ for the cases with (a) no void $(\ell=0)$ and (b) a cathodic interface with $\ell=50 \mu \mathrm{m}$ void. Results are shown for selected values of the normalised filament tip resistance $\kappa R_{\text {tip }}$ and an imposed current $\left|j_{\infty}\right|=i_{\text {CCD }}$. Time $t=0$ corresponds to the instant that the current was imposed.

\footnotetext{
${ }^{4}$ The presence of the void does not affect $b_{\mathrm{CCD}}$ with $b_{\mathrm{CCD}}$ only a function of $a_{0}$ for given electrolyte properties; see Eq. (2.11).
} 


\subsection{Influence of filament tip resistance on growth rates}

We now consider the effect of a finite value of $R_{\text {tip }}$ upon the growth rate of filaments. Predictions of filament length $a$ versus time $t$ are included in Figs. $6 \mathrm{a}$ and $6 \mathrm{~b}$ for the case of no void and $\ell=50 \mu \mathrm{m}$, respectively, for an imposed current $\left|j_{\infty}\right|=i_{\mathrm{CCD}}$ in each case. Results are shown for three selected values of $\kappa R_{\text {tip }}$ including the reference case of $R_{\text {tip }}=0$. Increasing the resistance for the flux of $\mathrm{Li}^{+}$through the filament tip can significantly reduce the filament growth rates. For example, for the $\ell=50 \mu \mathrm{m}$ case (Fig. 6b) the filament grows to $a=100 \mu \mathrm{m}$ in about $80 \mathrm{~s}$ with $R_{\text {tip }}=0$ compared to it taking $950 \mathrm{~s}$ with $\kappa R_{\text {tip }}=15$. An important consequence of the reduction in the filament growth rates with increasing $\kappa R_{\text {tip }}$ is that the fields within the electrolyte are less affected by filament growth than for the case shown in Fig. 5. In fact in this figure, we chose to show the distributions only for the high filament growth rates with $R_{\text {tip }}=0$ as the effect of filament growth is most clearly visible for that case. For the case with $\kappa R_{\text {tip }}=15$, the difference in overpotential between the stationary filament and the growing filament at the filament tip is less than $3 \%$ after the filament has grown from $a_{0}=$ $5 \mu \mathrm{m}$ to $a=11 \mu \mathrm{m}$.
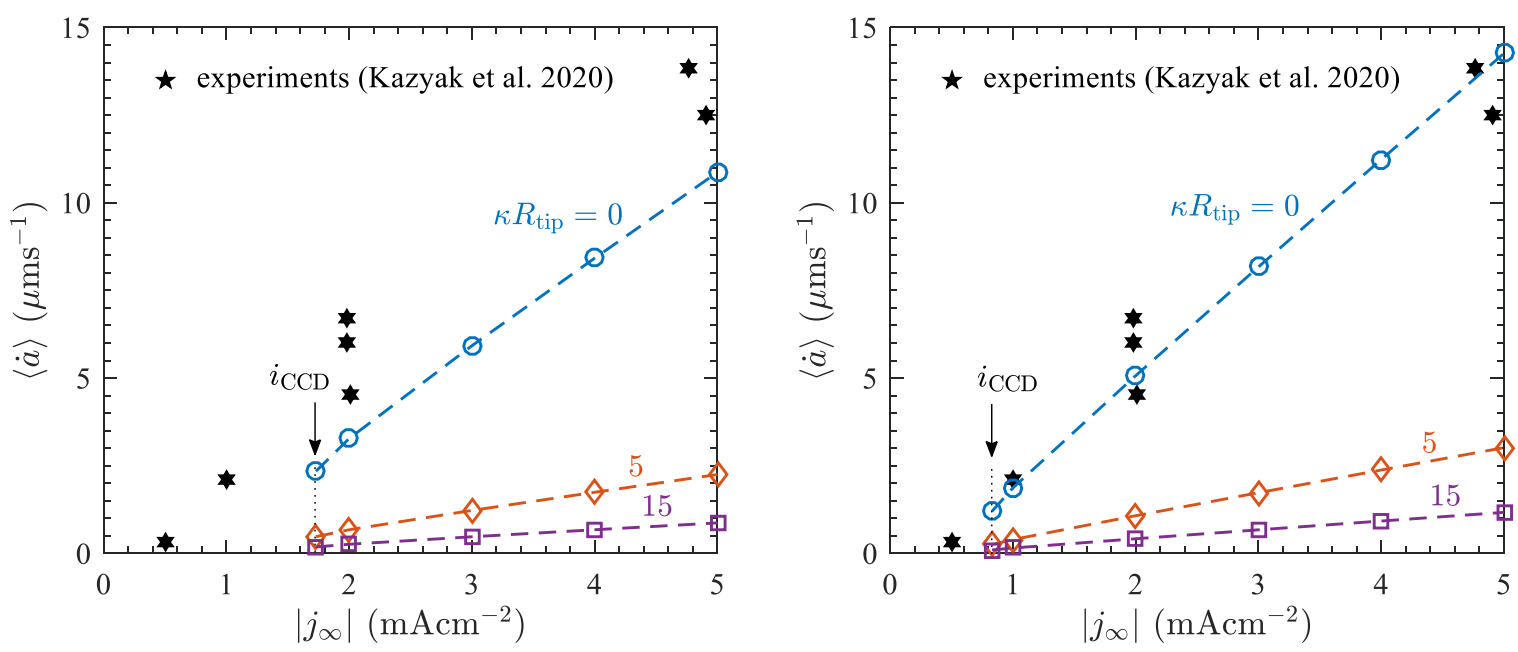

Figure 7: Comparisons between measurements [13] and predictions of the filament growth rates as a function of cell current $\left|j_{\infty}\right|>i_{\mathrm{CCD}}$ for three choices of the normalised filament tip resistance $\kappa R_{\mathrm{tip}}$. Results are shown for the cases with (a) no void $(\ell=0)$ and (b) a cathodic interface with $\ell=50 \mu \mathrm{m}$ void.

Direct measurements of filament growth rates in the literature are sparse: the authors are only aware of the measurements as reported in [13]. In this study, an "in-plane visualisation platform" was used to image and measure filament growth. In such a setup, both electrodes are deposited on the same surface of the electrolyte. This allows Li filaments to grow between the two electrodes and thus to be visualised side-on. The main difference to the situation analysed here, wherein the electrolyte is sandwiched between the two metal electrodes, is that the current in the "in-plane visualisation platform" is not spatially uniform around the growing filaments. Nevertheless, it is instructive to compare our predictions with these available measurements. Predictions of the average growth rate $\langle\dot{a}\rangle$ for filament growth from $a_{0}=5 \mu \mathrm{m}$ to $a_{0}=$ $100 \mu \mathrm{m}$ as a function of the cell current $\left|j_{\infty}\right|$ are included in Figs. $7 \mathrm{a}$ and $7 \mathrm{~b}$ for the case of no 
void and $\ell=50 \mu \mathrm{m}$, respectively. In each case, we include the computed values of $\langle\dot{a}\rangle$ for three choices of $\kappa R_{\text {tip }}$ for $\left|j_{\infty}\right|$ in the range $i_{\mathrm{CCD}}<\left|j_{\infty}\right| \leq 5 \mathrm{~mA} \mathrm{~cm}^{-2}$. The measurements from [13] are included with the current taken to be the nominal currents between the electrodes. In line with the measurements, the computed filament growth rates increase approximately linearly with $\left|j_{\infty}\right|$ and a comparison between predictions and measurements suggest that the case of the $\ell=50 \mu \mathrm{m}$ void with $R_{\text {tip }} \approx 0$ best correlates with the measurements. This strongly suggests that the resistance to the flux of $\mathrm{Li}^{+}$across the newly formed electrolyte surfaces at the filament tip is negligible; in contrast, the electrolyte/electrode interfaces possesses a finite resistance $Z$, presumably due to contamination of the pre-existing electrolyte surface. Kazyak et al. [13] come to a similar conclusion from a comparison of the response of filament/electrolyte interfaces to interfaces between deposited electrodes and electrolyte. The comparisons between measurements and predictions in Fig. 7 need to be viewed with caution as the electrical loading setup in the simulations does not precisely replicate the experiments.

\section{Concluding remarks}

Filaments modelled as edge dislocations grow by the flux of $\mathrm{Li}^{+}$into the filament tip at constant chemical potential. The growth rate is set by the balance of the loss of free-energy at the filament tip and the dissipation associated with the resistance to the flux of $\mathrm{Li}^{+}$through the filament tip. We model this process via a superposition scheme comprising the analyticallyknown infinite medium singular field of a sink of $\mathrm{Li}^{+}$located at the filament tip and a numerically evaluated smooth field that corrects for the boundary conditions of the electrolyte in the battery.

Numerical results are presented for (i) perfect interfaces between the electrolyte and electrodes such that the interfacial resistance is spatially uniform and (ii) a void is present along the electrolyte/cathode interface that prevents flux of $\mathrm{Li}^{+}$across that portion of the interface. While the presence of the void decreases the critical current density $i_{\mathrm{CCD}}$, the filament growth rates are lower by the presence of the void as the growth rates scale with the magnitude of the imposed currents. An increase in the resistance for flux of $\mathrm{Li}^{+}$into the filament tip reduces the filament growth rate for applied currents that exceed the critical current density. Consistent with recent measurements [13], the filament growth rates increase linearly with increasing cell current. These measurements also show that growth rates can be on the order of $10 \mu \mathrm{m} \mathrm{s}^{-1}$ and our calculations suggest that this implies a vanishing filament tip resistance $R_{\text {tip }}$. In line with observations [13], our calculations suggest that above the critical current density a filament can traverse the electrolyte in less than a few minutes indicating that the initiation of filament growth is the critical step in preventing short-circuiting of the battery.

\section{Appendix A: The shrinkage of the filament during the stripping phase}

Consider a filament of length $a$ as shown in Fig. A.1a emanating from the stripping electrode (anode) maintained at an electrical potential $\Phi_{\mathrm{P}}$ while the opposite plating electrode is taken to be ground, $\Phi=0$. The occupancy of Li sites within the filament is such that the chemical 
potential of the $\mathrm{Li}^{+}$within the filament is equal to that of the $\mathrm{Li}^{+}$in the adjacent electrolyte along the length $\xi$. We restrict ourselves to a mode whereby, similar to the growth of the filament, the retraction only occurs by flux of $\mathrm{Li}^{+}$out of the filament tip. Moreover, while flux of $\mathrm{Li}^{+}$is accompanied by elastic deformation of the electrolyte, we assume that an infinitesimally thin layer of Li remains so that the cracked ceramic does not reheal. Fluxing of $\mathrm{Li}^{+}$from the filament into the electrolyte occurs at constant chemical potential of $\mathrm{Li}^{+}$with electrons released due to this flux flowing through the external circuit to the cathode so as to maintain electrical neutrality. Moreover, the chemical potential of the electrons in the metal phase is also held fixed at $\mu_{\mathrm{el}^{-}}=-F \Phi_{\mathrm{P}}$ by the external circuit. We consider this to be an equilibrium process (at constant temperature, and chemical potentials $\mu_{\mathrm{tip}}$ and $\mu_{\mathrm{el}}{ }^{-} \mathrm{of} \mathrm{Li}^{+}$and electrons, respectively) and are therefore interested in the free-energy change associated with this process. This analysis follows closely to that developed in [18] and here we summarise the key steps.

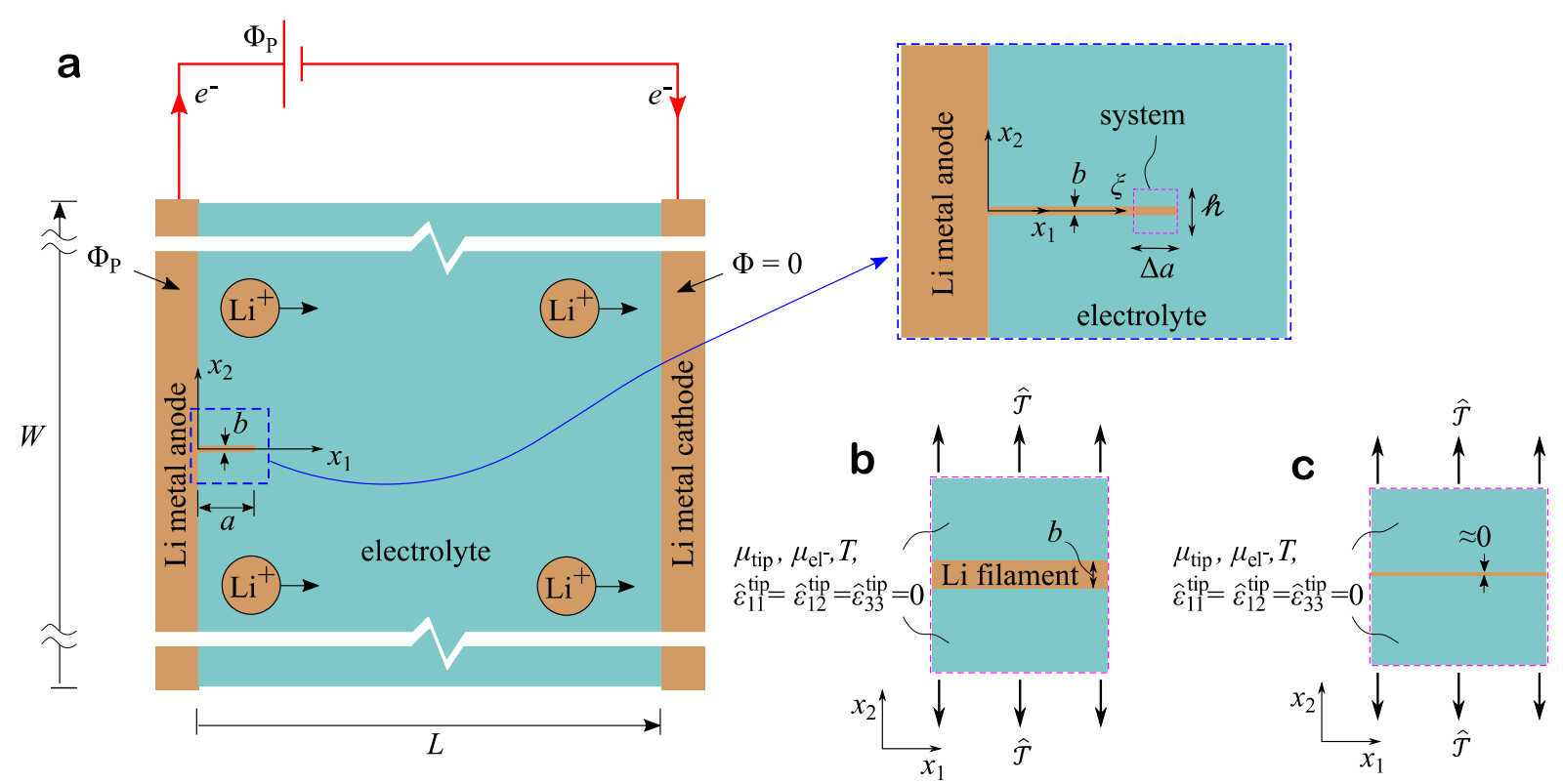

Figure A.1: (a) Sketch of a symmetric cell with a filament emanating from the anode. (b) A sketch of a system representing the electrolyte near the tip of the filament (see inset in (a)). The system comprising filament sandwiched between the electrolyte is maintained at a constant chemical potential $\mu_{\text {tip }}$ of $\mathrm{Li}^{+}$ and $\mu_{\mathrm{el}^{-}}$of electrons and the strains held fixed are labelled. (c) A sketch of the state of the system after retracting the filament through the system.

To calculate the change in free-energy of the system resulting from the filament retracting, we consider a spatially uniform system that is representative of a material point at the filament tip (Fig. A.1b where this is referred to as state A of the system). The system is of arbitrary dimensions and without loss of generality we shall set the system to be a cuboid of dimension $\Delta a \times h \times B$, where $h$ is a length measured perpendicular to the filament, $B$ is the thickness of the system in the $x_{3}$-direction and equal to the thickness of the electrolyte and the length of the system in the direction along the filament is set equal to the length $\Delta a$ by which we assume the filament retracts, i.e. $\Delta a$ is positive for decreasing $a$. The system is in equilibrium with a reservoir maintained at constant chemical potentials $\mu_{\text {tip }}$ and $\mu_{\mathrm{el}^{-}}{\mathrm{of} \mathrm{Li}^{+}}^{+}$and electrons, 
respectively, and temperature $T$. With $\phi_{\text {tip }}$ denoting the electric potential at the filament tip, this chemical potential is given by (2.1) and the occupancy of the $\theta_{\text {tip }}$ of lattice sites within the metal phase at the filament tip is given by a relation analogous to (2.4) as

$$
\frac{1}{\theta_{\text {tip }}}=1+\exp \left[\frac{F\left(\Phi_{\mathrm{P}}+\eta_{\text {tip }}\right)-h_{\mathrm{v}}}{R T}\right] \text {. }
$$

Now consider the retracted state of the filament (Fig. A.1c) with the system now in state B and the metal phase forming an infinitesimally thin layer. The system in state B comprises $N=$ $\rho_{\mathrm{m}} b B \Delta a$ fewer moles of Li compared to the system in state A, where $\rho_{\mathrm{m}}$ is the theoretical molar density of Li. Associated with this retraction is a change in the mechanical potential energy of the system given by $\Delta \Pi=-(\widehat{\mathcal{T}} B \Delta a) b$, where $\widehat{\mathcal{T}} \equiv-f_{\text {climb }} / b$ with

$$
f_{\text {climb }}=\frac{G b^{2}}{4 \pi(1-v) a} \text {. }
$$

To calculate the appropriate free-energy $\mathcal{L}_{\text {tip }}$ associated with the change in the state of the system from state A to state $\mathrm{B}$, recall that while the stress $\hat{\mathcal{T}}$, the chemical potential $\mu_{\text {tip }}$ of the $\mathrm{Li}^{+}$and the chemical potential $\mu_{\mathrm{el}^{-}}$of the electrons in the metal phase are held constant in the fracture process (i.e. need to be natural variables of $\mathcal{L}_{\text {tip }}$ ), the other natural variables are those of the Helmholtz free-energy of the system. Thus, taking the appropriate Legendre transforms, the free-energy of the system in state A with $N_{\mathrm{Li}^{+}}$moles of $\mathrm{Li}^{+}$and $N_{\mathrm{el}^{-}}$moles of electrons in the filament phase is given in terms of the Helmholtz free-energy $A_{\text {tip }}^{(\mathrm{A})}$ as

$$
\mathcal{L}_{\text {tip }}^{(\mathrm{A})}=A_{\text {tip }}^{(\mathrm{A})}-(\hat{\mathcal{T}} B \Delta a)\left(\hat{\varepsilon}_{22}^{\mathrm{tip}} h\right)-\mu_{\text {tip }} N_{\mathrm{Li}^{+}}-\mu_{\mathrm{el}^{-}} N_{\mathrm{el}^{-}},
$$

where

$$
\widehat{\mathcal{T}} \equiv \frac{1}{(B h \Delta a)} \frac{\partial A_{\mathrm{tip}}^{(\mathrm{A})}}{\partial \hat{\varepsilon}_{22}^{\mathrm{tip}}}, \quad \mu_{\mathrm{tip}} \equiv \frac{\partial A_{\mathrm{tip}}^{(\mathrm{A})}}{\partial N_{\mathrm{Li}^{+}}} \text {and } \mu_{\mathrm{el}^{-}} \equiv \frac{\partial A_{\mathrm{tip}}^{(\mathrm{A})}}{\partial N_{\mathrm{el}^{-}}}
$$

In (A.4), the partial derivatives are taken with all other natural variables of $A_{\text {tip }}^{(\mathrm{A})}$ held fixed while $\hat{\varepsilon}_{22}^{\text {tip }}$ is the strain of the electrolyte at the filament tip in the $x_{2}$-direction associated only with the image field of the dislocation; see [18] for details. In state B, the two halves of the fractured electrolyte are in the same state as in state A and the only differences between the two states are that state B has (i) an electroneutral metal phase comprising $N$ fewer moles of $\mathrm{Li}^{+}$and $N$ fewer moles of electrons and (ii) the system has strained by a nominal strain $-b / h$ in the $x_{2}$-direction with respect to state A. Then, analogous to (A.3) the free-energy of state $\mathrm{B}$ is

$$
\mathcal{L}_{\text {tip }}^{(\mathrm{B})}=\left(A_{\text {tip }}^{(\mathrm{A})}-A_{\mathrm{m}}\right)-(\hat{\mathcal{T}} B \Delta a)\left(\hat{\varepsilon}_{22}^{\mathrm{tip}} h-b\right)-\mu_{\mathrm{tip}}\left(N_{\mathrm{Li}^{+}}-N\right)-\mu_{\mathrm{el}}-\left(N_{\mathrm{el}^{-}}-N\right),
$$

where $A_{\mathrm{m}}$ is the Helmholtz free-energy of the metal phase in state A. This metal phase comprises Li atoms much like the electrodes but with a different occupancy of Li sites. Thus, using the fact that the total number of lattice sites in the metal phase is $N_{\mathrm{L}}^{\mathrm{m}}=N / \theta_{\text {tip }}$ with $\theta_{\text {tip }}$ given by (A.1), $A_{\mathrm{m}}$ follows from [18] as 


$$
A_{\mathrm{m}}=N \mu_{\mathrm{m}}^{0}+N\left(\frac{1}{\theta_{\text {tip }}}-1\right) h_{\mathrm{v}}-\left(\frac{N}{\theta_{\text {tip }}}\right) T s_{\text {tip }}
$$

where the specific entropy is

$$
s_{\text {tip }} \equiv-R\left[\theta_{\text {tip }} \ln \theta_{\text {tip }}+\left(1-\theta_{\text {tip }}\right) \ln \left(1-\theta_{\text {tip }}\right)\right] \text {. }
$$

Then, the change in free-energy of the system upon filament retraction is

$$
\Delta \mathcal{L}_{\text {tip }} \equiv \mathcal{L}_{\text {tip }}^{(\mathrm{B})}-\mathcal{L}_{\text {tip }}^{(\mathrm{A})}=(\widehat{\mathcal{T}} B \Delta a) b+N\left(\mu_{\text {tip }}+\mu_{\mathrm{el}^{-}}\right)-A_{\mathrm{m}} .
$$

Now, using the fact that $N=\rho_{\mathrm{m}} b B \Delta a$ along with the Eqs. (2.1), (A.2), $\mu_{\mathrm{el}^{-}}=-F \Phi_{\mathrm{P}}$ and the definition of the open circuit potential $\mathcal{U}$, i.e.

$$
\mu_{\mathrm{m}}^{0}+F \mathcal{U}=\mu_{\mathrm{e}}^{0}+R T \ln \left(\frac{\theta_{\mathrm{e}}^{0}}{1-\theta_{\mathrm{e}}^{0}}\right),
$$

it follows that

$$
\frac{\Delta \mathcal{L}_{\text {tip }}}{B \Delta a}=-\rho_{\mathrm{m}} b\left[F\left(\Phi_{\mathrm{P}}+\eta_{\text {tip }}\right)-T \frac{s_{\text {tip }}}{\theta_{\text {tip }}}+\left(\frac{1}{\theta_{\text {tip }}}-1\right) h_{\mathrm{v}}\right]-\frac{G b^{2}}{4 \pi(1-v) a},
$$

where $\eta_{\text {tip }} \equiv-\left(\phi_{\text {tip }}+\mathcal{U}\right)$. The practical case of $h_{\mathrm{v}} /(R T) \gg 1, \theta_{\text {tip }} \rightarrow 1$ and $s_{\text {tip }} \rightarrow 0$ leads to simplify (A.10) as

$$
\frac{\Delta \mathcal{L}_{\mathrm{tip}}}{B \Delta a} \approx-\left[\rho_{\mathrm{m}} b F\left(\Phi_{\mathrm{P}}+\eta_{\mathrm{tip}}\right)+\frac{G b^{2}}{4 \pi(1-v) a}\right] .
$$

The process of transforming the system from state A to B is spontaneous if $\Delta \mathcal{L}_{\text {tip }} \leq 0$. Assuming linearized Butler-Volmer kinetics, for the case of a stationary filament,

$$
\Phi_{\mathrm{P}}+\eta_{\text {tip }}=j_{\infty} Z\left(1+\frac{a}{\kappa Z}\right)
$$

where $j_{\infty}>0$ is the cell current. Thus, it follows from (A.11) that filament retraction with $\Delta \mathcal{L}_{\text {tip }}<0$ occurs for all $j_{\infty}$ (recall retraction corresponds to $\Delta a>0$ ). Thus, the filaments emanating from the anode retract so long as there exists an external circuit to complete the electron transfer from the anode to cathode.

The retraction rate can be computed in a manner analogous to that developed for the growth of the filament in Section 3. Define

$$
\eta_{\mathrm{c}}=-\left(\Phi_{\mathrm{P}}+\frac{G b}{F \rho_{\mathrm{m}} 4 \pi(1-v) a}\right)
$$

so recalling that $\Delta a$ is positive for retraction it follows from (A.11) that

$$
\dot{\mathcal{L}}_{\text {tip }}=-B v_{r} F \rho_{\mathrm{m}} b\left(\eta_{\text {tip }}-\eta_{\mathrm{c}}\right),
$$

where $v_{r}=-\dot{a}$ is the retraction rate. Using the definition (3.3) for the dissipation rate and recalling that $\dot{\mathcal{L}}_{\text {tip }} \leq 0$, we obtain

$$
v_{r}=\frac{1}{F \rho_{\mathrm{m}} b} \frac{\left(\eta_{\text {tip }}-\eta_{\mathrm{c}}\right)}{R_{\mathrm{tip}}}
$$


and the retraction rate can be calculated using the superposition scheme detailed in Section 3. The key difference, of course, is the fact that retraction occurs for all $j_{\infty}>0$ while the growth of the filament from the cathode, discussed in the main body of the paper, requires a critical current below which the filament is stationary.

\section{Acknowledgements}

The authors are grateful for helpful discussions with Profs. Peter Bruce, Clare Grey and Jeff Sakamoto. NAF acknowledges support by the ERC project "Multilat" and by the Faraday Institution [Solbat, grant number FIRG007].

\section{References}

[1] K. Takada, Progress and prospective of solid-state lithium batteries, Acta Mater. 2013, 61, 759-770.

[2] A. Hooper, B.C. Tofield, All-solid-state batteries, J. Power Sources 1984, 11, 33-41.

[3] L. Gireaud, S. Grugeon, S. Laruelle, B. Yriex, J.-M. Tarascon, Lithium metal stripping/plating mechanisms studies: A metallurgical approach, Electrochem. Commun. 2006, 8, 1639-1649.

[4] K.J. Harry, D.T. Hallinan, D.Y. Parkinson, A.A. MacDowell, Detection of subsurface structures underneath dendrites formed on cycled lithium metal electrodes, Nat. Mater. 2014, 13, 69-73.

[5] K.J. Harry, X. Liao, D.Y. Parkinson, A.M. Minor, N.P. Balsara, Electrochemical deposition and stripping behavior of lithium metal across a rigid block copolymer electrolyte membrane, $J$. Electrochem. Soc. 2015, 162, A2699-A2706.

[6] A. Sharafi, H.M. Meyer, J. Nanda, J. Wolfenstine, J. Sakamoto, Characterizing the $\mathrm{Li}-\mathrm{Li}_{7} \mathrm{La}_{3} \mathrm{Zr}_{2} \mathrm{O}_{12}$ interface stability and kinetics as a function of temperature and current density, J. Power Sources 2016, 302, 135-139.

[7] R.D. Schmidt, J. Sakamoto, In-situ, non-destructive acoustic characterization of solid state electrolyte cells, J. Power Sources 2016, 324, 126-133.

[8] A. Sharafi, E. Kazyak, A.L. Davis, S. Yu, T. Thompson, D.J. Siegel, N.P. Dasgupta, J. Sakamoto, Surface Chemistry Mechanism of Ultra-Low Interfacial Resistance in the Solid-State Electrolyte $\mathrm{Li}_{7} \mathrm{La}_{3} \mathrm{Zr}_{2} \mathrm{O}_{12}$, Chem. Mater. 2017, 29, 7961-7968.

[9] A. Sharafi, C.G Haslam, R.D. Kerns, J. Wolfenstine, J. Sakamoto, Controlling and correlating the effect of grain size with the mechanical and electrochemical properties of $\mathrm{Li}_{7} \mathrm{La}_{3} \mathrm{Zr}_{2} \mathrm{O}_{12}$ solid-state electrolyte, J Mater. Chem A 2017, 5, 21491.

[10] B. Wu, S. Wang, J. Lochala, D. Desrochers, B. Liu, W. Zang, J. Yang, J. Xiao, The role of the solid electrolyte interphase layer in preventing Li dendrite growth in solid-state batteries, Energy Environ. Sci. 2018, 11, 1803-1810.

[11] E.J. Cheng, A. Sharafi, J. Sakamoto, Intergranular Li metal propagation through polycrystalline $\mathrm{Li}_{6.25} \mathrm{Al}_{0.25} \mathrm{La}_{3} \mathrm{Zr}_{2} \mathrm{O}_{12}$ ceramic electrolyte, Electrochim. Acta 2017, 223, 85-91.

[12] L.C. De Jonghe, L. Feldman, A. Beuchele, Slow degradation and electron conduction in sodium/beta-aluminas, J. Mater. Sci. 1981, 16, 780-786. 
[13] E. Kazyak, R. Garcia-Mendez. W. S. LePage, A. Sharafi, A. L. Davis, A. J. Sanchez, K. Chen, C. Haslam, J. Sakamoto. N. P. Dasgupta, Li Penetration in Ceramic Solid Electrolytes: Operando Microscopy Analysis of Morphology, Propagation, and Reversibility, Matter 2020, 2, 1025-1048.

[14] L.A. Feldman, L.C. De Jonghe, Initiation of mode I degradation in sodium-beta alumina electrolytes, J. Mater. Sci. 1982, 17, 517-524.

[15] L. Porz, T. Swamy, B. W. Sheldon, D. Rettenwander, T. Frömling, H.L. Thaman, S. Berendts, R. Uecker, W. C. Carter, Y.-M. Chiang, Mechanism of Lithium Metal Penetration through Inorganic Solid Electrolytes, Adv. Energy Mater. 2017, 7, 1701003.

[16] M. Klinsmann, F.E. Hildebrand, M. Ganser, R.M. McMeeking, Dendritic cracking in solid electrolytes driven by lithium insertion, J. Power Sources 2019, 422, 227226.

[17] L. Barroso-Luque, Q. Tu, G. Ceder, Analysis of Solid-State Electrodeposition-InducedMetal Plastic Flow and Predictions of Stress States in Solid IonicConductor Defects, J. Electrochem. Soc. 2020, 167, 020534.

[18] S.S. Shishvan, N.A. Fleck, R.M. McMeeking, V.S. Deshpande, Dendrites as climbing dislocations in ceramic electrolytes: Initiation of growth, J. Power Sources 2020, 456, 227989.

[19] J. Kasemchainan, S. Zekoll, D.S. Jolly, Z. Ning, G.O. Hartley, J. Marrow, P.G. Bruce, Critical stripping current leads to dendrite formation on plating in lithium anode solid electrolyte cells, Nat. Mater. 2019, https://doi.org/10.1038/s41563-019-0438.

[20] C. D. Fincher, D Ojeda, Y. Zhang, G. M. Pharr, M. Pharr, Mechanical properties of metallic lithium: from nano to bulk scales, Acta Mater. 2020, 156, 215-222.

[21] A.J. Ilott, M. Mohammadi, H.J. Chang, C.P. Grey A. Jerschow, Real-time 3D imaging of microstructure growth in battery cells using indirect MRI, Proc. Natl. Acad. Sci. USA, 2016, 113, 10779-10784.

[22] H. Schultz, Defect parameters of bcc metals: group-specific trends, Mater. Sci. Eng. 1991, A141, 149-167.

[23] D. Rettenwander, A. Welzl, L. Cheng, J. Fleig, M. Musso, E. Suard, M.M. Doeff, G.J. Redhammer, G. Amthauer, Synthesis, Crystal Chemistry, and Electrochemical Properties of $\mathrm{Li}_{7-2 x} \mathrm{La}_{3} \mathrm{Zr}_{2-x} \mathrm{Mo}_{x} \mathrm{O}_{12}(x=0.1-0.4)$ : Stabilization of the Cubic Garnet Polymorph via Substitution of $\mathrm{Zr}^{4+}$ by $\mathrm{Mo}^{6+}$, Inorg. Chem. 2015, 54, 10440-10449.

[24] J.E. Ni, E.D. Case, J. Sakamoto, E. Rangasamy, J. Wolfenstine, Room temperature elastic moduli and Vickers hardness of hot-pressed LLZO cubic garnet, J. Mater. Sci. 2012, 47, 7978-7985.

[25] G. Papazafeiropoulos, M. Muniz-Calvente, E. Martínez-Pañeda, Abaqus2Matlab: A suitable tool for finite element post-processing, Adv. Eng. Software 2017, 105, 9-16. 